\title{
A NOVEL SHAPE FEATURE TO CLASSIFY MICROCALCIFICATIONS
}

\author{
By \\ Yiming Ma \\ A Thesis \\ Submitted to the \\ Faculty of the Graduate School \\ of \\ Western Carolina University \\ in Partial Fulfillment of \\ the Requirements for the Degree \\ of \\ Master of Science
}

Committee:

Director

Dean of the Graduate School

Date:

Spring 2010

Western Carolina University

Cullowhee, North Carolina 


\title{
A NOVEL SHAPE FEATURE TO CLASSIFY MICROCALCIFICATIONS
}

\begin{abstract}
A thesis presented to the faculty of the Graduate School of Western Carolina University in partial fulfillment of the requirements for the degree of Master of Science.
\end{abstract}

\author{
By \\ Yiming Ma \\ Director: Peter C. Tay, PhD \\ Assistant Professor \\ Department of Engineering and Technology
}

March 2010

(C) 2010 by Yiming Ma 
This thesis is dedicated to my parents. 


\section{ACKNOWLEDGEMENTS}

Dr. Peter C. Tay has been the ideal advisor. Without his sage advice for the research and patient criticism on composing final paper, this thesis would not have been possible.

I am grateful for having Dr. James Z. Zhang and Dr. Robert D. Adams as my committee member and their generous help in every aspect during the past two years.

Lastly, I offer my regards and blessings to all of my friends and classmates who supported me in any respect during the completion of the project. 


\section{TABLE OF CONTENTS}

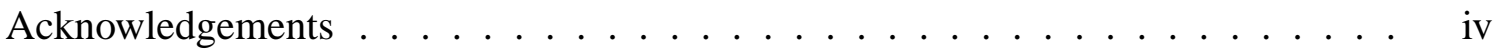

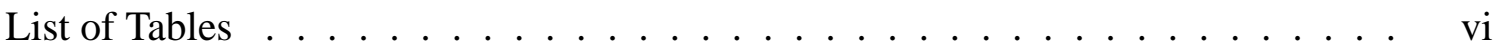

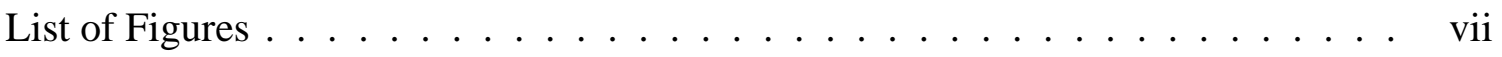

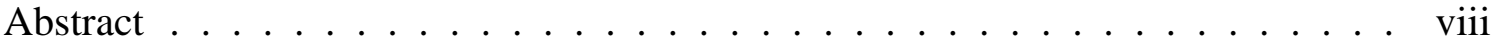

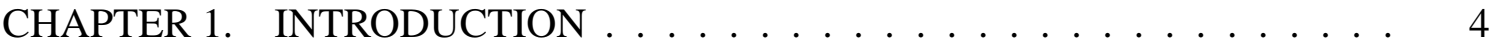

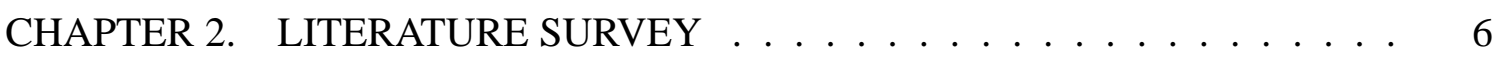

2.1 Haralick Feature . . . . . . . . . . . . . . . . . . . 7

2.2 Wavelet Feature . . . . . . . . . . . . . . . . 7

2.3 Shape Feature . . . . . . . . . . . . . . . 8

2.4 Other Features . . . . . . . . . . . . . . . . . . . . 10

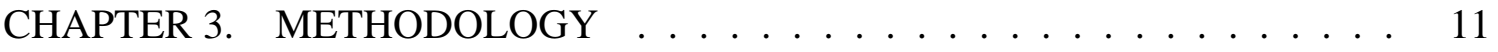

3.1 Region Growing Algorithm . . . . . . . . . . . . . . . . . 11

3.2 Gradient Vector Flow Active Contour . . . . . . . . . . . . . 12

3.3 A Normalized Distance Signature . . . . . . . . . . . . . . . 13

3.4 A Novel Roughness Metric . . . . . . . . . . . . . . . . . . . . 14

CHAPTER 4. EXPERIMENTATION AND RESULT . . . . . . . . . . . 20

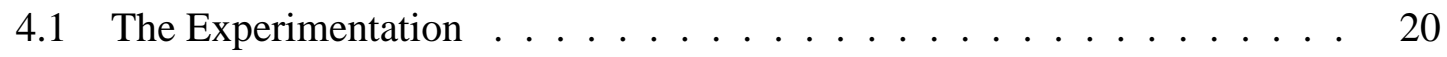

4.2 Receiver Operating Characteristic $\ldots \ldots \ldots \ldots \ldots . \ldots . \ldots 22$

4.3 Test Result . . . . . . . . . . . . . . . . . . . . . . . . 25

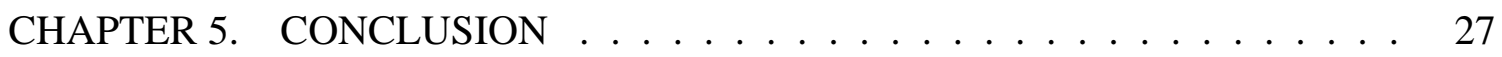

APPENDIX A. An example of the DDSM data . . . . . . . . . . . 30

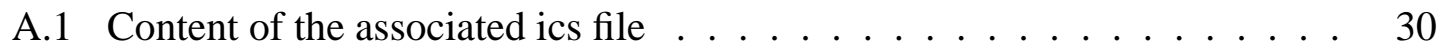

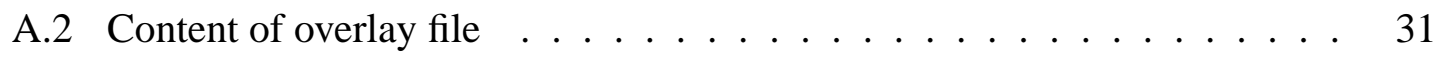

A.3 The image data . . . . . . . . . . . . . . . 32

APPENDIX B. Microcalcification with maximum $\alpha$ value for each ROI . . . . 34

APPENDIX C. Test result of all Microcalcifications . . . . . . . . . . . 40

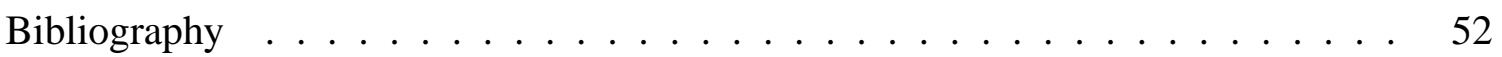




\section{LIST OF TABLES}

1.1 Estimated New Female Breast Cancer Cases and Deaths by Age, US, 2009

2.1 Table of Haralick features . . . . . . . . . . . . . . . . . . . 7

2.2 Table of shape features provided in $[1] \ldots \ldots \ldots$

3.1 Previously published and proposed $\alpha$ features of the contours shown in Fig. $3.4 \ldots \ldots \ldots \ldots$. . . . . . . . . . . . . . . . . . . . . .

4.1 Numbers of each lesion types in DDSM . . . . . . . . . . . . . 20

4.2 Ratios of benign cases . . . . . . . . . . . . . . . . . . 21

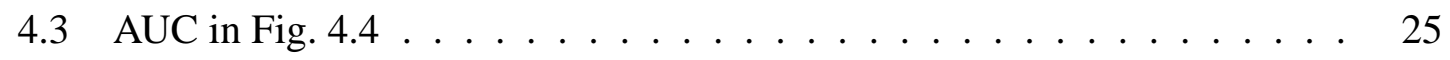

A.1 An example of the DDSM data . . . . . . . . . . . . 30

B.1 Microcalcification with maximum $\alpha$ value for each ROI . . . . . . . . . . 34

C.1 Test result of all Microcalcifications _. . . . . . . . . . . . . 40 


\section{LIST OF FIGURES}

3.1 An example of the RGM. The yellow pixels are the result of the region growing segmentation. . . . . . . . . . . . . . 12

3.2 An example of active coutour . . . . . . . . . . . . . . . . . 13

3.3 Wavelet decomposition of the normalized distance signature $\widetilde{\text { dist }}$ and reconstruction from only the $\mathbf{D}_{3}$ coefficients. . . . . . . . . . . . 15

3.4 Example of an ellipse, a circle, and a star contours. . . . . . . . . . . 16

3.5 The normalized distance signatures $(\widetilde{\mathbf{d i s t}}) \ldots \ldots \ldots$. . . . . . . . . 17

3.6 Approximations of the normalized distance signatures in Fig. 3.5 from using only the $\mathbf{D}_{3}$ wavelet coefficients. . . . . . . . . . . . . . 18

3.7 Flowchart of the proposed method . . . . . . . . . . . . . . 19

4.1 Relations between TPR, FPR, FNR and TNR . . . . . . . . . . . 22

4.2 An example of distributions to illustrate TPR and FPR. These distribution are not actual. . . . . . . . . . . . . . . . 23

4.3 A simulated ROC curve . . . . . . . . . . . . . . . . . . 24

4.4 ROC of the proposed method, compactness, moment, Fourier descriptor . 26

A.1 An example of a DDSM data . . . . . . . . . . . . 33 


\section{ABSTRACT}

\section{A NOVEL SHAPE FEATURE TO CLASSIFY MICROCALCIFICATIONS}

Yiming Ma, M.S.T.

Western Carolina University (March 2010)

Director: Peter C. Tay, PhD

Clinical evident shows that the shape of mammographic calcification is an indicator of the pathology. Microcalcifications (MC) with rough shape are early signs of malignant breast cancer. This thesis proposed a shape metric to help radiologist in classifying regions of interest. Region growing and gradient vector flow algorithm are used to obtain the contour of $\mathrm{MC}$ to calculate the normalized distance signature. A three level wavelet decomposition with a Daubechies eight tap wavelet is used to provide a bandpass function and extract the desired shape feature of the MC. A comparison with previously used shape features such as compactness, moment, Fourier descriptors is provided. 58 malignant and 125 benign cases, totaling 368 individual MC, are tested by the proposed method and previously used shape features. 


\section{CHAPTER 1: INTRODUCTION}

In 2006 cancer was reported as the second leading cause of death in the United States with 559,888 cases, which is equivalent to $23.1 \%$ of total deaths [2]. ${ }^{1}$ In [3], the American Cancer Society (ACS) reported that breast cancer is the most common cancer among women, accounting for nearly 1 in 4 cancers diagnosed in US women. ${ }^{2}$ Table. 1.1 shows ACS's most recent estimates [3] for breast cancer in the United States for $2009^{3}$ :

Table 1.1: Estimated New Female Breast Cancer Cases and Deaths by Age, US, 2009

\begin{tabular}{lccc} 
Age & In Situ Case & Invasive Cases & Deaths \\
\hline Younger than 45 & 6,460 & 18,640 & 2,820 \\
45 and older & 55,820 & 173,730 & 37,350 \\
Younger than 55 & 24,450 & 62,520 & 8,890 \\
55 and older & 37,830 & 129,850 & 31,280 \\
Younger than 65 & 40,940 & 120,540 & 17,200 \\
65 and older & 21,340 & 71,830 & 22,970 \\
\hline All Age & 62,280 & 192,370 & 40,170 \\
\hline
\end{tabular}

It is widely accepted that early detection tests for breast cancer can save many thousands of lives each year [4]. Mammography and palpation are the two conventional ways to screen for breast cancer. Mammography is the process of using X-rays radiation to examine the human breast and is used as a diagnostic as well as a screening tool. The

\footnotetext{
${ }^{1}$ Heart disease is listed as the leading cause with 631,636 cases, which is equivalent to $26.0 \%$ of total deaths in [2].

${ }^{2}$ Men are generally at low risk for developing breast cancer

${ }^{3}$ Rounded to the nearest 10
} 
presence of clusters of microcalcifications (MC), which are at least three tiny deposits of calcium within a one $\mathrm{cm}^{2}$ region, could provide information for experienced radiologist to detect the early sign of breast cancer [5]. MC generally have a diameter from $0.1 \mathrm{~mm}$ to $0.5 \mathrm{~mm}$ and are hard to detect, especially in dense parenchynmal tissue. The presence of MC or MC cluster does not necessarily indicate the presence of malignant cancer. Radiologists evaluate the shape, morphology, and pattern of clusters of MC to determine if a biopsy is warranted.

This paper presents a method to extract a new shape feature from MC found in mammography. The background of computer-aided-analysis and previous work are discussed in Chapter 2; the proposed method is presented in detail in Chapter 3; a comparison of the proposed method with the previous classifier is presented in Chapter 4. Chapter 5 presents a conclusion. 


\section{CHAPTER 2: LITERATURE SURVEY}

There are variety of proposed methods to classify mammographic region of interests (ROIs). A summary of previous methods used in various stages of computer-aided diagnosis (CAD) are provided. Although the following is not a fully inclusive list, the described features represent a wide range of MC characteristics. Several features such as Haralick features, wavelet features, multi-wavelet features and shape feature [1] are developed as classifier for MC and MC cluster. Individual MC features, statistical texture features, multi-scale texture features as well as fractal dimension features are used in detecting MC [6]. Segmentation process usually takes place together with or after successful detection. The purpose of segmentation is to separate certain featured parts from each other and the background of an image and to divide those into non-overlapping regions. The two main reasons for MC segmentation are to obtain the location of MC to assist radiologists and to classify the abnormalities into benign and malignant categories [7]. In mammography analysis, the background is not uniformly changing due to the nonuniformity of the density of breast tissue. Therefore segmentation of MC usually demands an adaptive method. MC usually appear brighter than its background. If a local threshold method is used to detect MC, then a threshold value for a sub-image and a selection of window size are required. Arikidis et al. present a size-adapted MC segmentation by applying a multiple scale active contour, which is initialized by active ray [8]. Other methods such as non-linear filter, wavelet transform [9] active contour [10] and neutral network [11] have also been proposed. Also in $[12,13]$, the authors proposed another approach to CAD 
mammography by detecting a whole MC cluster rather than a single MC.

\subsection{Haralick Feature}

The Haralick feature analysis $[14,15]$ is known as the spatial gray-level dependence method. This method has previously been used for MC classification in MRI images. The basis for these features is the gray-level co-occurrence matrix $\mathrm{H}$ given by

$$
\mathbf{H}=\left[\begin{array}{cccc}
P(1,1) & P(1,2) & \ldots & P\left(1, N_{0}\right) \\
P(2,1) & P(2,2) & \ldots & P\left(2, N_{0}\right) \\
\vdots & \vdots & \ddots & \vdots \\
P\left(N_{0}, 1\right) & P\left(N_{0}, 2\right) & \ldots & P\left(N_{0}, N_{0}\right)
\end{array}\right]
$$

where $P(i, j)$ is defined as the number of $i^{\text {th }}$ pixel values that are adjacent to the $j^{\text {th }}$ pixel

Table 2.1: Table of Haralick features

\begin{tabular}{ll}
\hline Angular Second Moment & Contrast \\
Corelation & Sum of Squares: Variance \\
Inverse Difference Moment & Sum Average \\
Sum Variance & Sum Entropy \\
Entropy & Difference Variance \\
Difference Entropy & Info. Measure of Correlation 1 \\
Info. Measure of Correlation 2 & Max. Correlation Coefficient \\
\hline
\end{tabular}

value, divided by the total number of such comparisons. The dimension of this matrix is $N_{0} \times N_{0}$, where $N_{0}$ is the number of gray levels in the image. Each entry represents the probability that an $i^{\text {th }}$ pixel value is adjacent to a $j^{\text {th }}$ pixel value. Based on this gray-level co-occurrence matrix $\mathbf{H}$, Haralick generated 14 texture features listed in Table 2.1.

\subsection{Wavelet Feature}

Another widely used method is analyzing an image in multi-scale resolution. In [16], a general paradigm for the analysis and application of discrete multiwavelet transforms 
is provided. Wavelet analysis is a powerful tool to achieve multi-scale resoloution. A computer-aided method to overcome the two main difficulties of detecting MC, which are low contrast between MC and its background and high false positive rate in MC classification, is provided in [17] with applying wavelet to remove the background noise and Markov random field to enhance the recognition of MC.

The extraction of wavelet feature is based on decomposing the image and calculating the entropy and energy of each sub-bands. In a $N \times N$ sub-image, normalized energy and entropy are computed as [1]:

$$
\begin{gathered}
\text { normalized energy }=\frac{\sum_{i} \sum_{j} x_{i j}}{N^{2}} \\
\text { normalized entropy }=-\frac{\sum_{i} \sum_{j}\left[\frac{x_{i j}^{2}}{P^{2}}\right] \log _{2}\left[\frac{x_{i j}^{2}}{P^{2}}\right]}{\log _{2} N^{2}}
\end{gathered}
$$

where $x_{i j}$ is the $i^{\text {th }}, j^{\text {th }}$ pixel value of the sub-images, and

$$
P^{2}=\sum_{i} \sum_{j} x_{i j}^{2}
$$

\subsection{Shape Feature}

In [18], the authors proposed a combination of three shape features of individual MC shape and claimed a $100 \%$ accuracy of detection by applying a nearest-neighbor method classifier. The mathematical expressions of these three features are:

\section{Compactness}

$$
C=\frac{P^{2}}{A}
$$

where $P$ is the length of region perimeter and $A$ is the area of the MC. 
2. Moment $F_{3}-F_{1}$

Given $z(i), i=1,2, \ldots, N$ are the Euclidean distances of the ordered contour pixels to the reference (centroid) point and $N$ is the number of contour pixels, the $p^{\text {th }}$ moment is defined as

$$
m_{p}=\frac{1}{N} \sum_{i=1}^{N}|z(i)|^{p} .
$$

The $p^{\text {th }}$ central moment is defined as

$$
M_{p}=\frac{1}{N} \sum_{i=1}^{N}\left|z(i)-m_{1}\right|^{p}
$$

The $F_{3}-F_{1}$ feature is used as the shape feature for classifying $\mathrm{MC}$ and is defined as

$$
F_{3}-F_{1}=\frac{M_{4}^{\frac{1}{4}}}{m_{1}}-\frac{M_{2}^{\frac{1}{2}}}{m_{1}} .
$$

\section{Fourier Descriptor}

The Fourier Descriptor is computed by representing each pixel by a complex number $Z_{i}=x_{i}+j y_{i}$, where $x_{i}$ and $y_{i}$ are the coordinate of each pixels. The Fourier descriptors are defined as:

$$
A(n)=\frac{1}{N} \sum_{i=0}^{N-1} Z_{i} e^{-j \frac{2 \pi}{N} n i}
$$

To make the Fourier descriptor invariant to position, size, orientation and starting point of the contour, the Fourier Descriptor is normalized as:

$$
N F D(k)=\left\{\begin{array}{ll}
0 & k=0 \\
A(k) / A(1) & k=1,2, \ldots, N / 2 \\
A(k+N) / A(1) & k=-1,-2, \ldots,-N / 2+1
\end{array} .\right.
$$

The feature FF (Fourier Feature) is defined based on the NFD as:

$$
F F=\frac{\sum_{k=-N / 2+1}^{N / 2}\|N F D(k)\| /|k|}{\sum_{k=-N / 2+1}^{N / 2}\|N F D(k)\|} .
$$

where $\|N F D(k)\|$ denotes the magnitude of $N F D(k)$. 


\subsection{Other Features}

Table 2.2: Table of shape features provided in [1]

\begin{tabular}{cl}
\hline 1 & Number of microcalcifications in cluster \\
2 & Maximum size of microcalcifications in cluster \\
3 & Standard deviation of the size of microcalcifications in cluster \\
4 & Number of microcalcifications with size of one pixel \\
5 & Sum of the area of the microcalcifications in each cluster \\
6 & Maximum value of compactness in cluster \\
7 & Average compactness in cluster \\
8 & Maximum value of F3-F1 in cluster \\
9 & Average value of F3 in cluster \\
10 & Maximum value of F3 in cluster \\
11 & Average F3 in cluster \\
12 & Radius of the circle that best fits the cluster \\
13 & Scattering of the microcalcifications \\
14 & Average gray level of the microcalcifications in cluster \\
15 & Standard deviation of the mean of the microcalcification gray levels in the cluster \\
16 & Maximum standard deviation of the gray levels in each microcalcification \\
17 & Average standard deviation of the gray levels in each calcification in cluster \\
\hline
\end{tabular}

The features described in this chapter are not a fully complete list. The reader is referred to [1] for a more comprehensive description of the proposed $\mathrm{MC}$ feature listed in Table 2.2. 


\section{CHAPTER 3: METHODOLOGY}

This chapter provides detailed information about the proposed method to classify benign and malignant cases by analyzing the MC shape feature. Firstly, the definition and attribute of the distance signature are introduced. Secondly, explanation of why and how I implemented the algorithm is presented. Finally, the flow chart of the complete method is given in Fig 3.7.

\subsection{Region Growing Algorithm}

The region growing method (RGM) is adopted to segment MC in my proposed method. The RGM starts from a user specified seed point with a chosen tolerance $(0 \leq \tau \leq 1$, typically $\tau=0.025)$. Initially, the seed point is the only point of a region. A neighboring point value $p(i, j)$ of a region is added to the region if the following criteria is satisfied:

$$
(1-\tau) \frac{\left(P_{\max }+P_{\min }\right)}{2} \leq p(i, j) \leq(1+\tau) \frac{\left(P_{\max }+P_{\min }\right)}{2}
$$

where $P_{\max }$ and $P_{\min }$ is the maximum and minimum value of the current region and $\tau$ is a predefined tolerance. Each iteration grows the region until a maximum number of iterations is performed. The maximum search iteration in my proposed method is set to 500. It has been empirically verified that this depth is sufficient enough for the size of MC being analyzed. An example of the results of region growing with tolerance $\tau=0.025$ is shown as yellow within a radiologist defined ROI (outlined in red) in Fig. 3.1. 


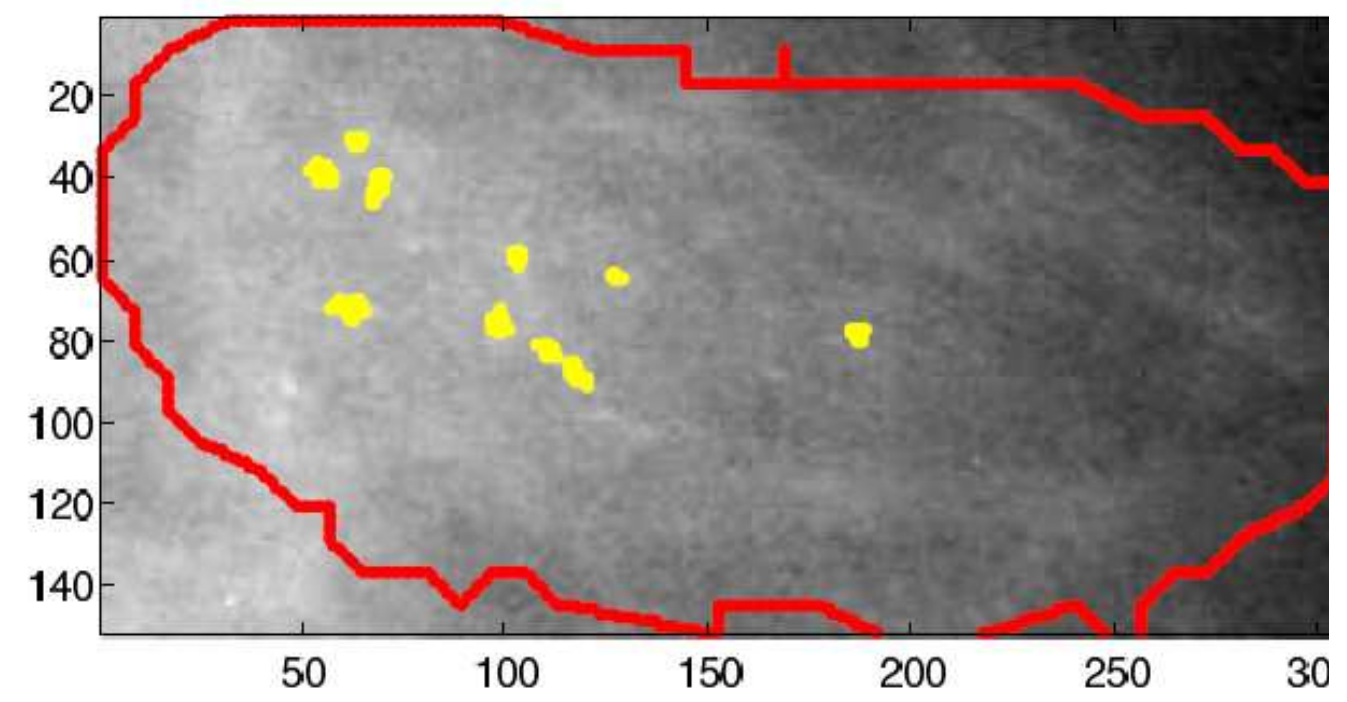

Figure 3.1: An example of the RGM. The yellow pixels are the result of the region growing segmentation.

\subsection{Gradient Vector Flow Active Contour}

The gradient vector flow (GVF) active contour, or snake [19] is used to get an order set of close contour points from the region growing results. Active contours are widely used in computer vision and image processing applications. Particularly it is used to outline boundaries of an object or other desired features within an image [20]. The snakes are curves defined within an image domain, which could move according to the internal force and the external gradient field until finally reaching a balanced state. Since the external gradient field is computed from the image data, the snake will conform to the boundaries of the object or other desired image feature. An example of active contour is provided in Fig. 3.2 [19]. The dynamic snake equation is defined in equation (3.2):

$$
X(s, t)=\alpha X^{\prime \prime}(s, t)-\beta X^{\prime}(s, t)+V(x, y)
$$

where $\mathbf{X}(s, t)=[x(s, t), y(s, t)]$ is the snake and $s \in[0,1], \mathbf{X}^{\prime}$ and $\mathbf{X}^{\prime \prime}$ denote the first and second derivatives of $\mathbf{X}$ with respect to $s, \alpha$ and $\beta$ are weighting parameters applied to 
Deformation in progress, iter $=125$

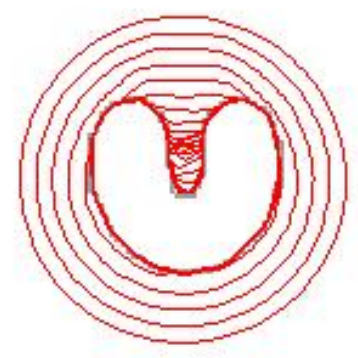

edge map gradient

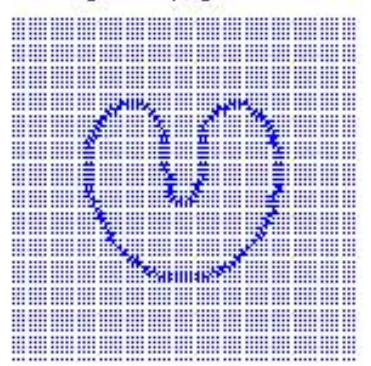

edge map

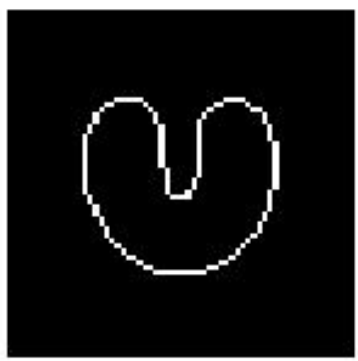

normalized GVF field

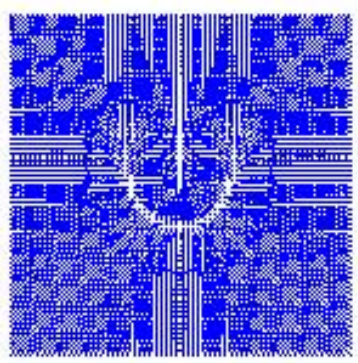

Figure 3.2: An example of active coutour

the snakes tension and rigidity, respectively. The last term of equation (3.2) is $V(x, y)=$ $[u(x, y), v(x, y)]$, the gradient vector field that minimized the energy functional $\varepsilon$, defined in equation (3.3)

$$
\varepsilon=\iint \mu\left(u_{x}^{2}+u_{y}^{2}+v_{x}^{2}+v_{y}^{2}\right)+|\nabla f|^{2}|V(x, y)-\nabla f|^{2} d x d y
$$

where $f(x, y)$ is the edge map and $\mu$ is a parameter governing the tradeoff between the first term and the second term in the integrand.

\subsection{A Normalized Distance Signature}

The signature of a shape is used extensively in image processing shape analysis applications. The shape signature can be generated in various ways [21]. In [18], an order set 
of close contour points of each MC within a radiologist defined ROI is required to extract shape features. A normalized distance signature is extracted from the order set of close contour from GVF contour described in the previous section of this chapter. The distance signature is defined as the Euclidean distance of the ordered contour point to the centroid [18] in equation (3.4)

$$
\operatorname{dist}[i]=\sqrt{\left(x_{i}-x_{c}\right)^{2}+\left(y_{i}-y_{c}\right)^{2}} \text {. }
$$

It should be noted that this distance signature is invariant to various affine transformations like translations and rotations. The distance signature of equation (3.4) will change with the size of an object. The scale variance is partly due to the digitizing error of the coordinate of the contour point, which in most of the cases has a negligible effect. Another reason why this signature is only pseudo-scale invariance is that a length of the normalized signature in equation (3.5) varies with respect to the size of the object and sampling of the contour. To make it pseudo-invariant to scale, the distance signature is normalized by dividing the mean. The normalized distance signature used in the proposed feature is

$$
\widetilde{\operatorname{dist}}[i]=\frac{\operatorname{dist}[i]}{\operatorname{mean}(\operatorname{dist})} .
$$

This normalization provides a pseudo-scale invariance to the distance signature.

\subsection{A Novel Roughness Metric}

The novel aspect of the proposed method is using frequency analysis of the normalized distance signature to evaluate the roughness of a MC contour. Bandpass filtering is provided by a three level wavelet decomposition and reconstruction with a Daubechies eight tap wavelet. The reconstruction using only the third detail wavelet coefficients $\mathbf{D}_{3}$ is used to compute the roughness feature. In Fig. 3.3, the filtering processes is illustrated by a 
block diagram. The filters $F_{a}(z), G_{a}(z), F_{s}(z)$, and $G_{s}(z)$ denote the $z$-transforms of the eight tap Daubechies analysis scaling function, analysis wavelet, synthesis scaling function, and synthesis wavelet, respectively. The processes denoted by $\downarrow 2$ and $\uparrow 2$ indicate down sampling and up sampling by a factor of two, respectively.

An example using a circle, an ellipse and a star shaped contour is displayed in Fig. 3.4.

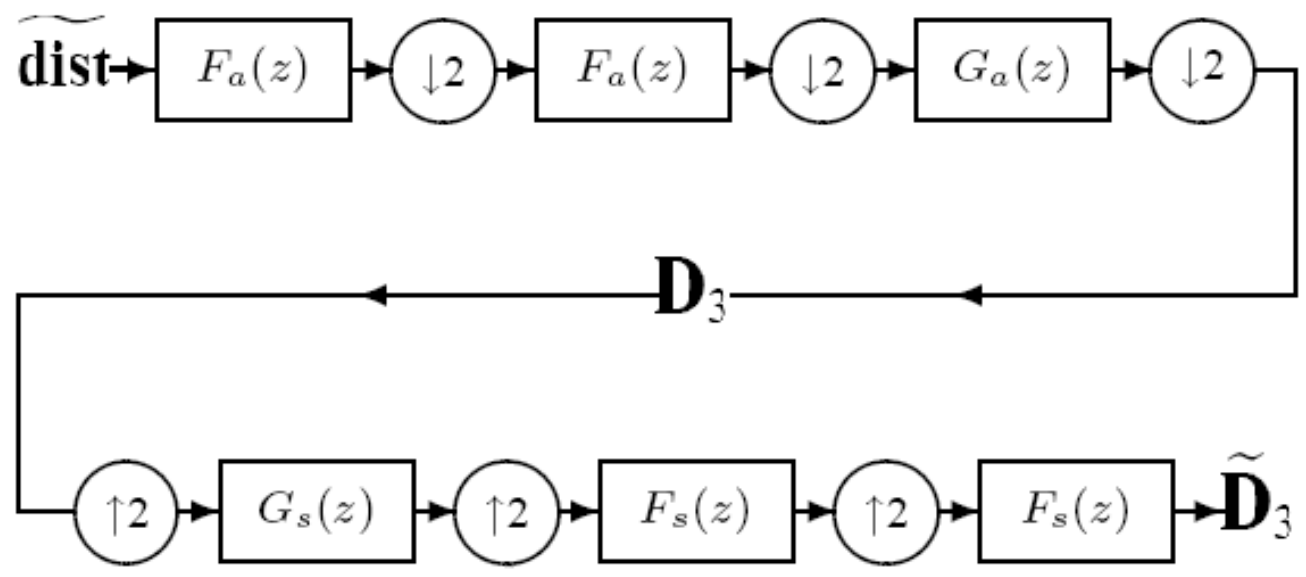

Figure 3.3: Wavelet decomposition of the normalized distance signature $\widetilde{\text { dist }}$ and reconstruction from only the $\mathbf{D}_{3}$ coefficients.

The normalized distance signatures of circle, ellipse and star are shown in Fig. 3.5 as the green, blue, and red plots, respectively. The performance of the proposed metric is demonstrated by analyzing these three shapes. The filtering result of the normalized distance signatures is shown in Fig. 3.6.

Low frequency oscillations of the normalized distance signature indicate smoothness of the analyzed contour. It is shown by Fig. 3.5 that the normalized signature of the circle is constant and the star shaped contour has significantly higher frequency. To extract this higher frequency component of each normalized distance signature, reconstruction with only the $\widetilde{\mathbf{D}}_{3}$ coefficient of the normalized distance signature is employed. Fig. 3.6 shows the $\widetilde{\mathbf{D}}_{3}$ coefficient reconstruction of the circle, ellipse and star as the green, blue, 


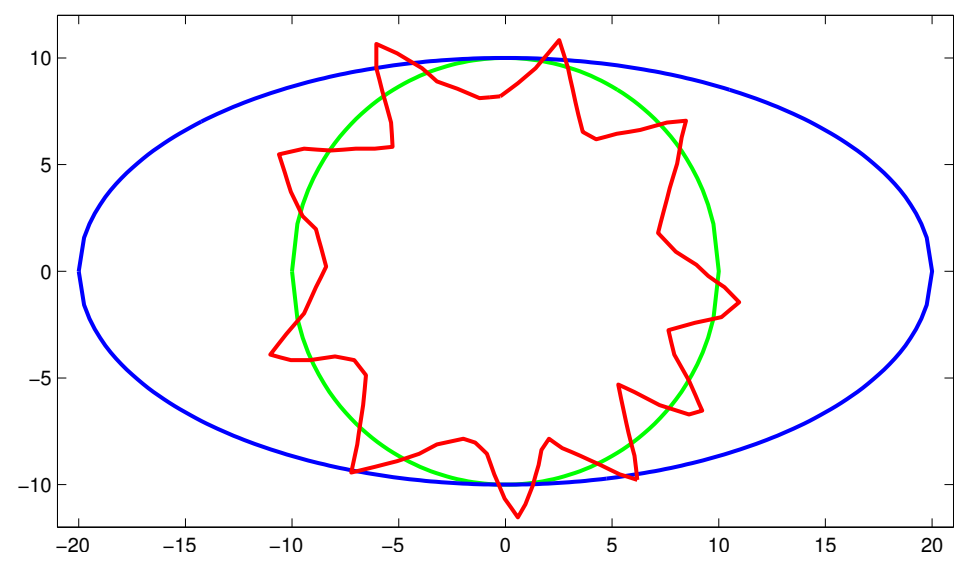

Figure 3.4: Example of an ellipse, a circle, and a star contours.

and red plots, respectively. A larger maximum absolute value of the $\widetilde{\mathbf{D}}_{3}$ reconstruction in Fig. 3.6 indicates a rougher contour. Therefore, the proposed novel metric $\alpha$ to classify $\mathrm{MC}$ is the maximum absolute value of reconstruction from the $\mathbf{D}_{3}$ coefficients

$$
\alpha=\max \left(\left|\widetilde{\mathbf{D}}_{3}\right|\right)
$$

The reason why absolute value is used as the shape feature is that a value in the reconstruction of $\widetilde{\mathbf{D}}_{3}$ would indicate a local difference. Whether this local difference will cause a positive or negative peak in the reconstruction of $\widetilde{\mathbf{D}}_{3}$ is dependent on how the filters are implemented. Also, a larger amplitude of the peak in the reconstruction of $\widetilde{\mathbf{D}}_{3}$ is attained by larger local differences. Further the absolute value makes the metric invariant to reflecting the contour about some line. A list of the roughness value $\alpha$ defined in equation (3.6) for the circle, ellipse, and the star shaped contour, as well as analysis using previously proposed shape feature is provided in Table 3.1. From Table 3.1, the roughness metric $\alpha$ has a desired relation that is substantially larger for the rough star shaped $(0.1528)$ contour than for the circle (0) and the ellipse (0.0095).

A flow chart of my shape analysis method is shown in Fig. 3.7. 


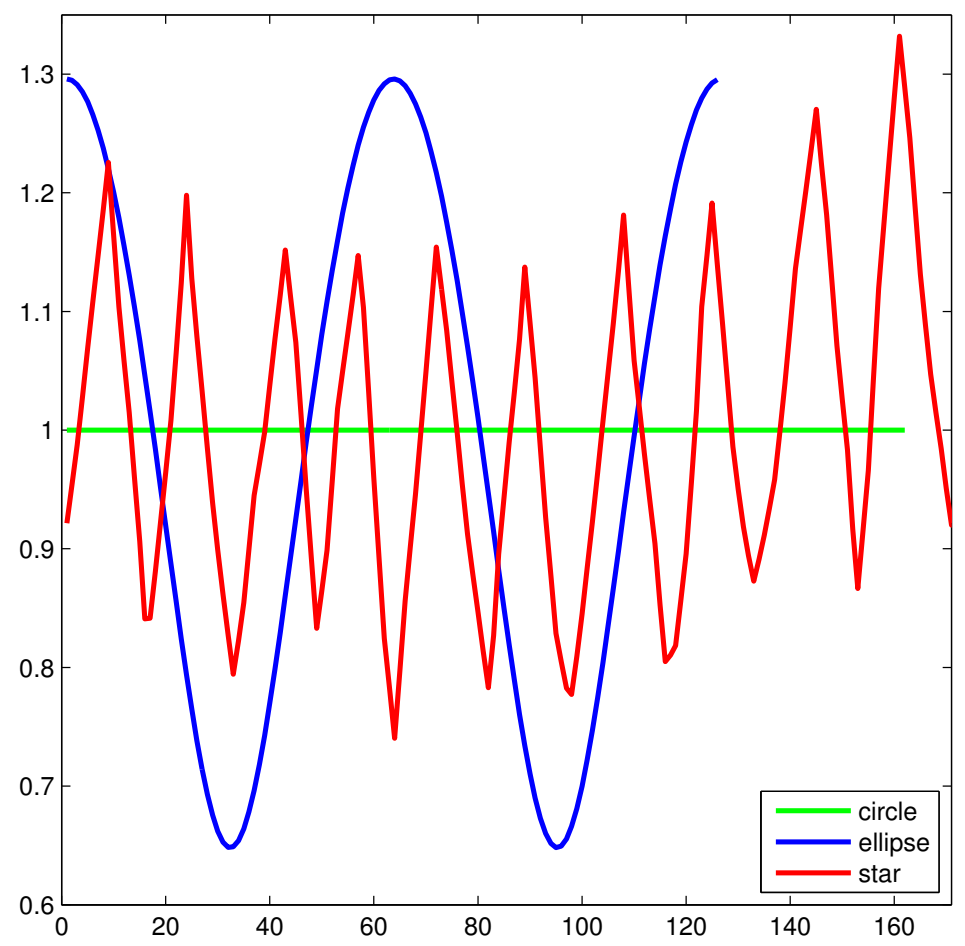

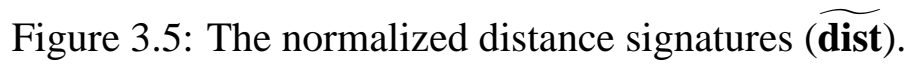

Table 3.1: Previously published and proposed $\alpha$ features of the contours shown in Fig. 3.4

\begin{tabular}{|c|c|c|c|c|}
\hline Contour & Moments & Fourier descriptor & Compactness & $\alpha$ \\
\hline circle & 0 & 0.1166 & 12.5571 & 0 \\
\hline ellipse & 0.0377 & 0.1215 & 14.9301 & 0.0095 \\
\hline star & 0.0297 & 0.0587 & 29.136 & 0.1528 \\
\hline
\end{tabular}




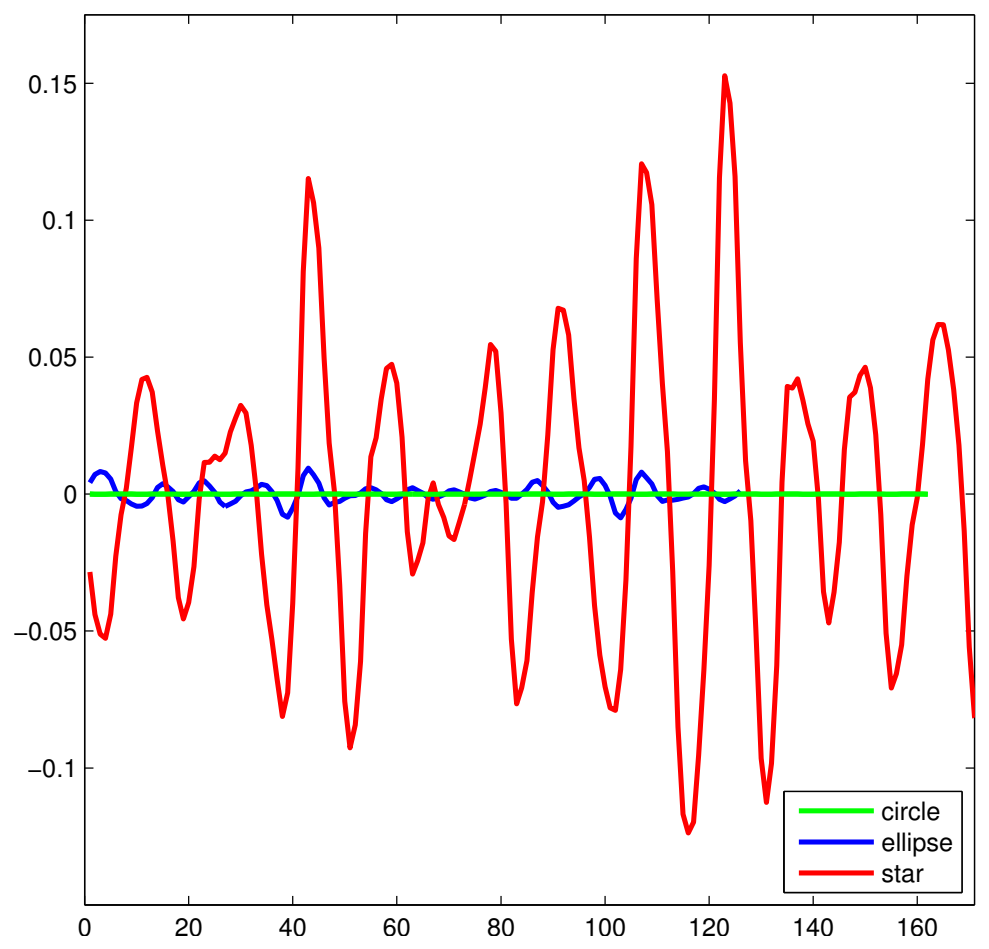

Figure 3.6: Approximations of the normalized distance signatures in Fig. 3.5 from using only the $\mathbf{D}_{3}$ wavelet coefficients. 


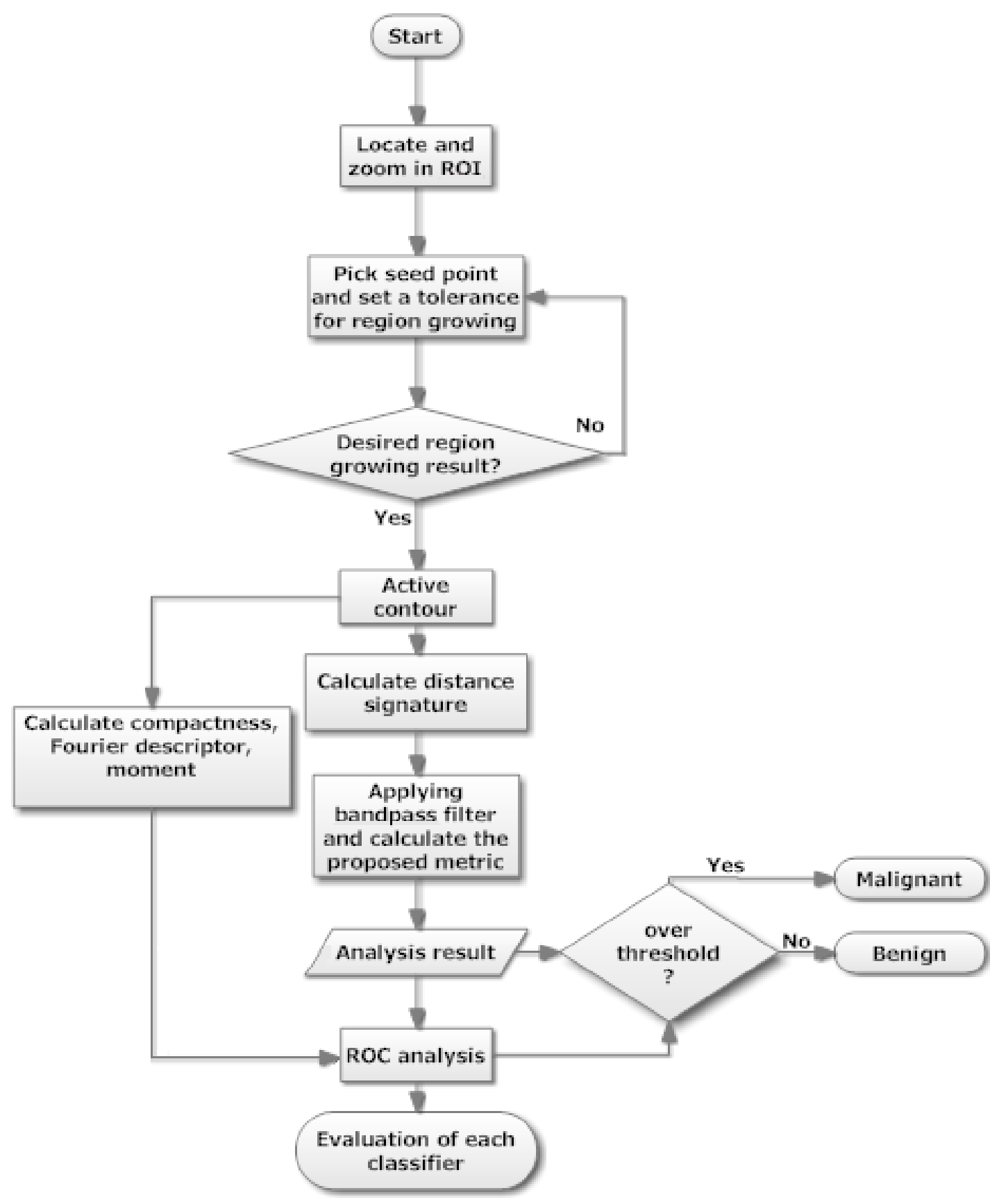

Figure 3.7: Flowchart of the proposed method 


\section{CHAPTER 4: EXPERIMENTATION AND RESULT}

An experiment was performed to evaluate the robustness of my proposed roughness metric. The experiment tested my proposed metric against three previously published shape metrics.

\subsection{The Experimentation}

A data mining of DDSM shows that mammograms with certain characteristics are problematic to diagnosis. The number of each lesion type in DDSM and the ratios of benign cases in each lesion type are listed in Table 4.1 and Table 4.2, respectively. In Table 4.2,

Table 4.1: Numbers of each lesion types in DDSM

\begin{tabular}{|l|c|c|c|c|c|}
\hline Type $\backslash$ Distribution & Cluster & Linear & Segmental & Regional & Diffuse \\
\hline Punctate & 82 & 4 & 20 & 6 & 4 \\
\hline Amorphous & 135 & 6 & 33 & 11 & 4 \\
\hline Pleomorphic & 716 & 70 & 140 & 27 & 0 \\
\hline Round \& regular & 8 & 0 & 0 & 0 & 0 \\
\hline Luscent center & 0 & 1 & 0 & 0 & 0 \\
\hline Fine 2 branching & 28 & 36 & 19 & 2 & 0 \\
\hline
\end{tabular}

the ratio of benign to total number of pleomorphic and cluster MC cases is close to 0.5 . This indicates that it is difficult to classify mammograms with this type of lesion. An example of a DDSM case of pleomorphic and clustered mammogram with associate data file is included in Appendix A. Other cases that also have ratio of benign cases close to 0.5 
Table 4.2: Ratios of benign cases

\begin{tabular}{|l|c|c|c|c|c|}
\hline Type $\backslash$ Distribution & Cluster & Linear & Segmental & Regional & Diffuse \\
\hline Punctate & 0.65 & 1.0 & 0.8 & 0.6 & 1.0 \\
\hline Amorphous & 0.79 & 0.3 & 0.79 & 0.45 & 0.5 \\
\hline Pleomorphic & 0.59 & 0.17 & 0.3 & 0.2 & N/A \\
\hline Round \& regular & 0.75 & N/A & N/A & N/A & N/A \\
\hline Luscent center & N/A & 1.0 & N/A & N/A & N/A \\
\hline Fine 2 branching & 0.18 & 0.25 & 0 & 1.0 & N/A \\
\hline
\end{tabular}

are ignored due to the small number of total cases.

The experiment was performed using 183 DDSM pleomorphic and clustered MC cases, from DDSM. This set included 58 malignant and 125 benign cases. There were a total of 368 individual MC that were analyzed. All 183 cases had only one radiologist defined ROI associated with each mammogram. The sole ROI contained BIRADS $\AA$ pleomorphic and clustered MC and biopsy was recommended in all cases. Only MC from the ROI were analyzed. The robustness of the segmentation of each MC was supervised by setting the tolerance parameter by trial and error so that a robust segmentation was attained. The GVF algorithms was also supervised to ensure that the contour of each MC was correctly captured. The initial curve of GVF was a small circle centered at the centroid of the MC determined by the region growing algorithm. In [5], a radiologist prescribe that a cluster can be classified as benign when all MC within the ROI are round or oval. Thus, a single rough shaped MC could indicate the development of malignant cancer. Therefore the maximum $\alpha$ attained by a selected MC in a ROI is used to classify the ROI as benign or malignant. The shape features of compactness, moment and Fourier descriptor, as described in [18] are used as a comparison to test improvements provided by my shape feature. 


\subsection{Receiver Operating Characteristic}

The receiver operating characteristic (ROC) graph is a technique to visualize the sensitivity and specificity of the performance of a certain classifier. It has been used in signal detection theory to describe the relation of hit rate and false alarm rate [22]. ROC is widely used to evaluate medical decision algorithms. The true positive rate (TPR), also called hit rate, represent the probability that a system could correctly classify given it is actually positive. The false positive rate (FPR), also called false alarm rate, indicates how often the system classify an actual negative as a positive. True positive rate (TPR) and false positive rate

\section{actual value}

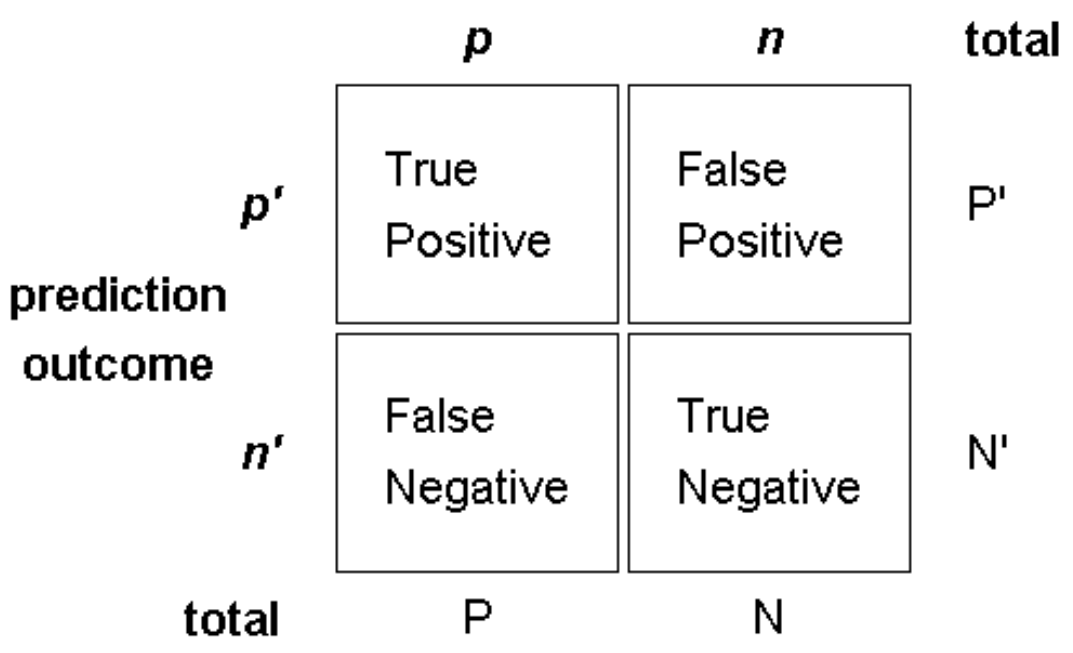

Figure 4.1: Relations between TPR, FPR, FNR and TNR

(FPR) are defined in equation (4.1) and equation (4.2) respectively, where TP is number of true positives, FN is number of false negatives, FP is number of false positives and TN is true negatives. The TPR and FPR are mathematically defined as

$$
T P R=\frac{T P}{T P+F N}
$$




$$
F P R=\frac{F P}{F P+T N}
$$

To show the significance of the ROC, let's assume benign MC have a shape feature, whose values have a Gaussian distribution with $\mu=1$ and $\sigma^{2}=0.49$, while the same feature of malignant MC have a Gaussian distribution with $\mu=-1$ and $\sigma^{2}=0.49$ as shown in the left plot of Fig. 4.2. If we choose 0.5 as the threshold of this classifier to determine the pathology, the TPR would be represented by the shaded part in the center plot of Fig. 4.2 while the FPR is represented by the shaded part in the right plot of Fig. 4.2. Though we
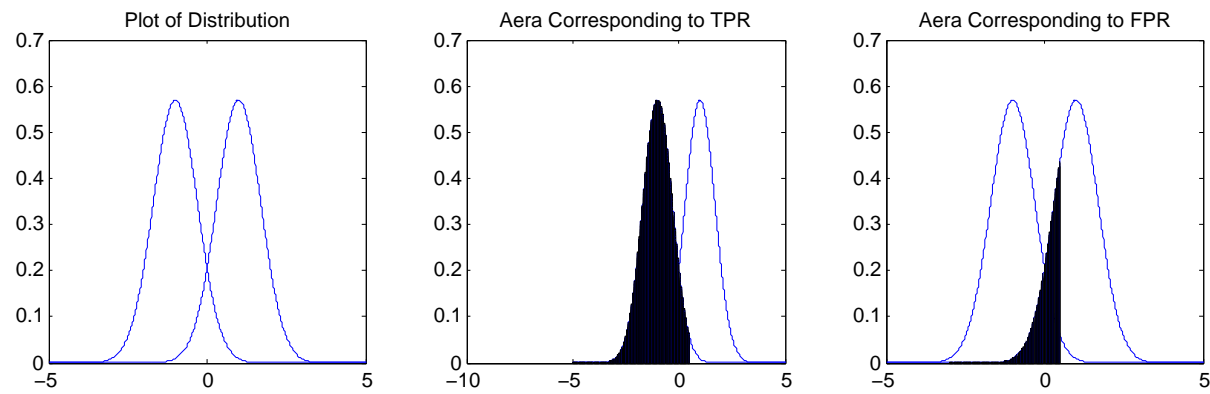

Figure 4.2: An example of distributions to illustrate TPR and FPR. These distribution are not actual.

could choose a different threshold to achieve a desired TPR or FPR, performance of the classifier is dependent on the overlap of the distributions. The ROC graph displays the dependence of the threshold on FPR and TPR. FPR is indicated on the x-axis and TPR is indicated on the y-axis. Fig. 4.3 shows an example of a ROC curve. It is widely quantified that a classifier with higher AUC (area under curve) is considered a better one, because it could achieve a higher TPR than one with lower AUC at the same FNR. The ROC curve and AUC are used to judge the performance of a classifier. It's ideal to get a $100 \%$ TPR with a $0 \%$ FPR. But in reality there is a trade off between these two. A simulated ROC curve is plotted in Fig. 4.3, if the threshold corresponding to the circle point on the ROC in Fig. 4.3 is used, we could achieve 35\% FNR and 55\% TPR performance. 


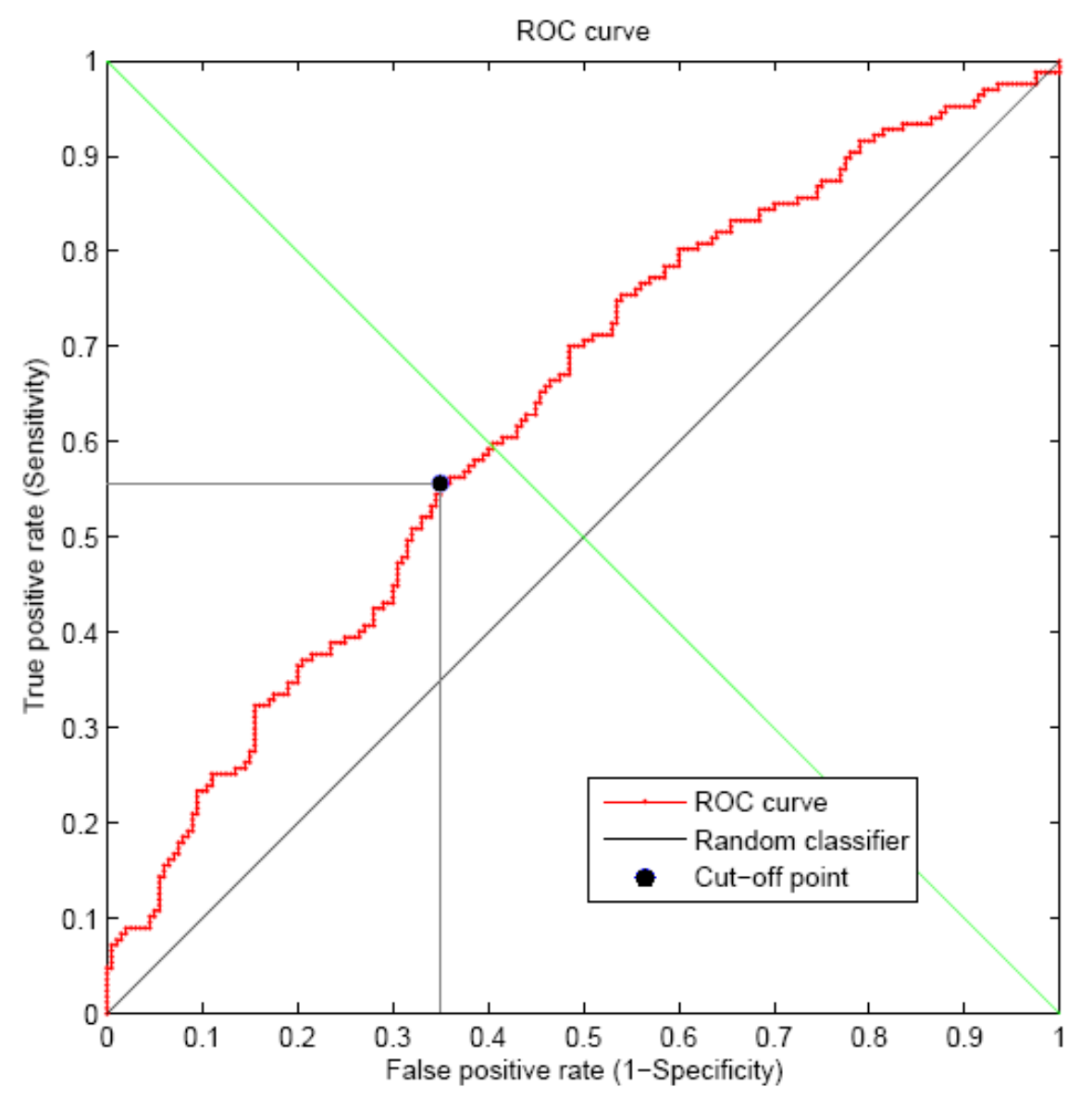

Figure 4.3: A simulated ROC curve 
Table 4.3: AUC in Fig. 4.4

\begin{tabular}{|c|c|}
\hline Feature & AUC \% \\
\hline Proposed $\alpha$ & 96.4 \\
\hline Moment & 78.0 \\
\hline Fourier descriptor & 73.9 \\
\hline Compactness & 56.5 \\
\hline
\end{tabular}

\subsection{Test Result}

The shape feature of each of the 368 MCs was computed using my proposed method to produce an $\alpha$ value, along with the Fourier descriptor, moment and the compactness shape feature. These computed shape feature measures are listed in Table C.1. Only the maximum $\alpha$ from each ROI was used to classify the pathology. Appendix B lists the computed shape feature measures for the MC which has the maximum value in each ROI. The F, FF and $\mathrm{C}$ data in Appendix B were used to generate the ROC and calculate the AUC for those features. The ROC of the proposed a measure, along with the ROCs of the moment, Fourier, and compactness measures are shown in Fig. 4.4. The corresponding AUC of each these four ROCs are listed Table 4.3. The ROC of my proposed shape measure shows better diagnostic accuracy than the compactness, moment and Fourier descriptor measures. The ROI of the $\alpha$ measure has a higher TPR at a cost of lower FPR as shown in Fig. 4.4. The AUC of my proposed metric is significantly higher than the other three.

Since missing a suspicious malignant case could be fatal, achieving a 100\% TPR is the priority in setting the threshold for the classifier. With the threshold for the proposed method set at $\alpha=02522$, all the malignant ROIs are correctly identified with a $20 \%$ FPR. Thus, it would have save $80 \%$ of the suspicious but benign cases from biopsy. Therefore $80 \%$ (100 out of 125) of the tested cases were correctly identified as benign, and all 58 tested malignant cases were correctly identified. 


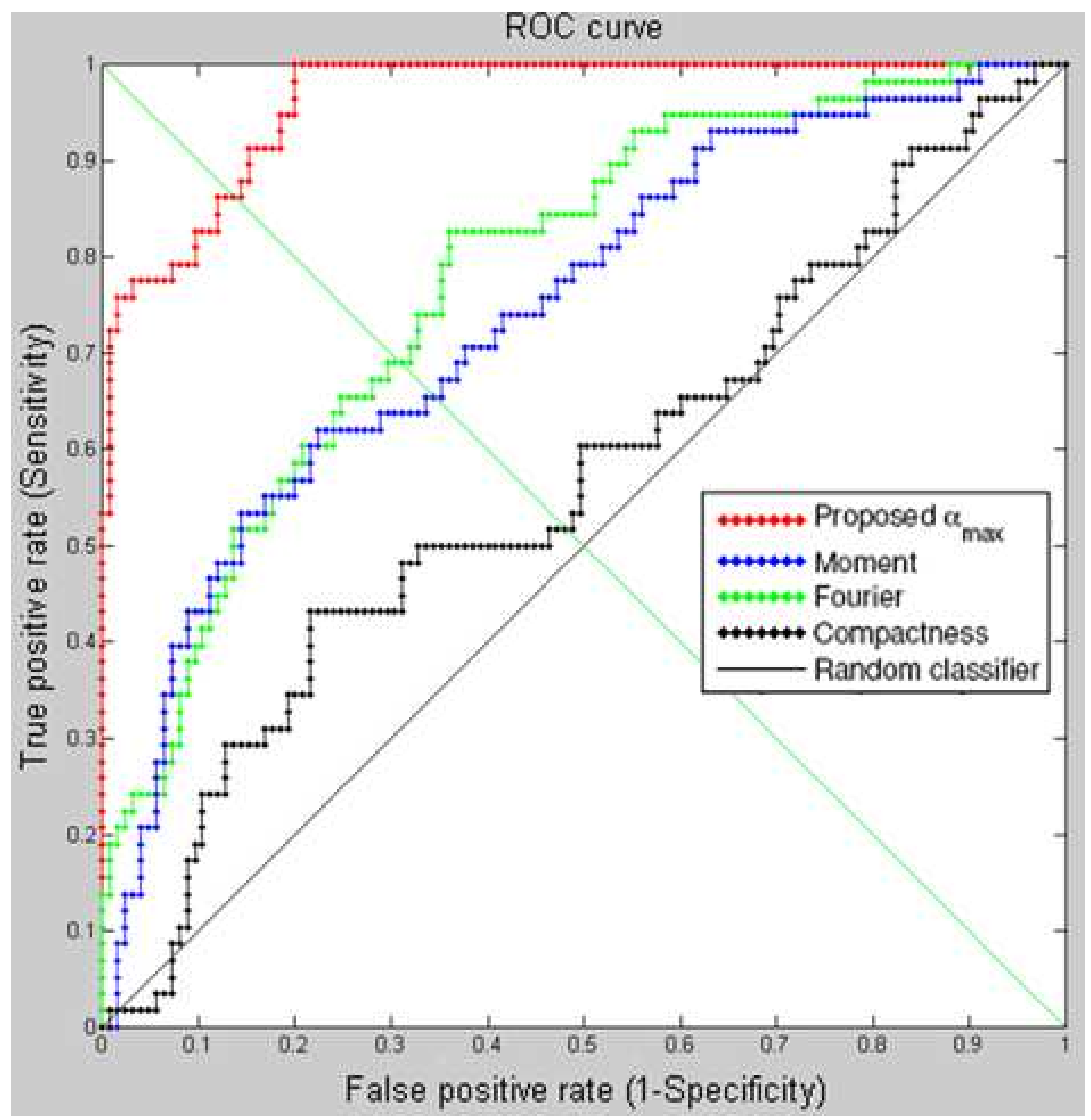

Figure 4.4: ROC of the proposed method, compactness, moment, Fourier descriptor 


\section{CHAPTER 5: CONCLUSION}

A computer aided method to assist radiologists in classifying mammograms with pleomorphic and clustered MC as well as other hard to diagnosis cases is presented in this thesis. As discussed in Chapter 1, it is standard to use mammography to detect early signs of breast cancer. Data mining of DDSM shows that ROIs with pleomorphic and clustered MC is problematic for radiologist to diagnose. Clinical evidence shows that ROIs containing tiny MC that are all round and oval have less of a chance in developing into malignant cancer [5]. Based on Sickles' claim in [5], an irregularly rough shaped MC could be an early sign of potentially malignant cancer.

The proposed method starts with a region growing process to segment the selected $\mathrm{MC}$, followed by the generation of a GVF active contour to define the outer contour of ordered points of each segmented MC. Then a distance signature sequence is generated from the Euclidean distance of each contour points to the centroid. After normalizing the distance signature, a novel feature $\alpha$ to represent roughness of the shape is extracted by bandpass filtering and full resolution reconstruction of the normalized distance signature. A shape feature $\alpha$ is computed from the filtered sequence is produced by the maximum absolute value of the bandpass approximation of the normalized distance signature using only the $\mathbf{D}_{3}$ wavelet coefficients. High $\alpha$ values indicate rough shaped contours. An evaluation using ROC and AUC was performed. It was shown that the maximum $\alpha$ attain by a MC of each ROI was a better classifier of malignant or benign than the individual Fourier descriptor, moment and compactness features. Finally a threshold value for $\alpha$ is set by 
ROC analysis to achieve $100 \%$ TPR at a low cost of $20 \%$ FPR. Therefore, the experimentation in this thesis provides evidence that my proposed shape analysis and metric is better classifier than any of the three previously published shape metrics. 
Appendices 


\section{APPENDIX A: AN EXAMPLE OF THE DDSM DATA}

Table A.1: An example of the DDSM data

\begin{tabular}{|l|c|}
\hline Pathology & Benign \\
\hline Case No. & 1265 \\
\hline Machine & HOWTEK \\
\hline filename & A_1265_1.RIGHT_MLO \\
\hline overlay file & A_1265_1.RIGHT_MLO.OVERLAY \\
\hline
\end{tabular}

A.1 Content of the associated ics file

ics_version 1.0

filename A-1265-1

DATE_OF_STUDY 2511995

PATIENT_AGE 48

FILM

FILM_TYPE REGULAR

DENSITY 3

DATE_DIGITIZED 2371998

DIGITIZER HOWTEK 43.5

SEQUENCE

LEFT_CC LINES 6556 PIXELS_PER_LINE 4111 BITS_PER_PIXEL 12 RESOLUTION 43.5 NON_OVERLAY 
LEFT_MLO LINES 6616 PIXELS_PER_LINE 4081 BITS_PER_PIXEL 12 RESOLUTION 43.5 NON_OVERLAY

RIGHT_CC LINES 6646 PIXELS_PER_LINE 4066 BITS_PER_PIXEL 12 RESOLUTION 43.5 OVERLAY

RIGHT_MLO LINES 6871 PIXELS_PER_LINE 3961 BITS_PER_PIXEL 12 RESOLUTION 43.5 OVERLAY

\section{A.2 Content of overlay file}

TOTAL_ABNORMALITIES 1

ABNORMALITY 1

LESION_TYPE CALCIFICATION TYPE PLEOMORPHIC DISTRIBUTION CLUSTERED

LESION_TYPE MASS SHAPE IRREGULAR MARGINS ILL_DEFINED

ASSESSMENT 4

SUBTLETY 3

PATHOLOGY BENIGN

TOTAL_OUTLINES 1

BOUNDARY

99961650000066666666666666000006666677777666660000 666600000666660000066666000006666677777666660000 066666666600006666666666000006666666666777706666 600000666660000066666000006666600007777700000000 000000000000222220000000002222200000111112222211 111222222222222220000022222222222222222220000222 222222222222222222222222222222222222222222222222 
224444222223333322222444442222222222444442222222

223333322222444442222222222444433334222224444422 222444444444422222444444444444444444455555444444 444466666444444444666664444466665555566666444446 666644444666664444666664444466666666644444666666 $666655555 \#$

\section{A.3 The image data}

MC_BEN_1265 right medio-latheral raw pixel is displayed as a gray scale image in Fig. A.1. The circle in Fig A.1 is the ROI assigned by a radiologist. 


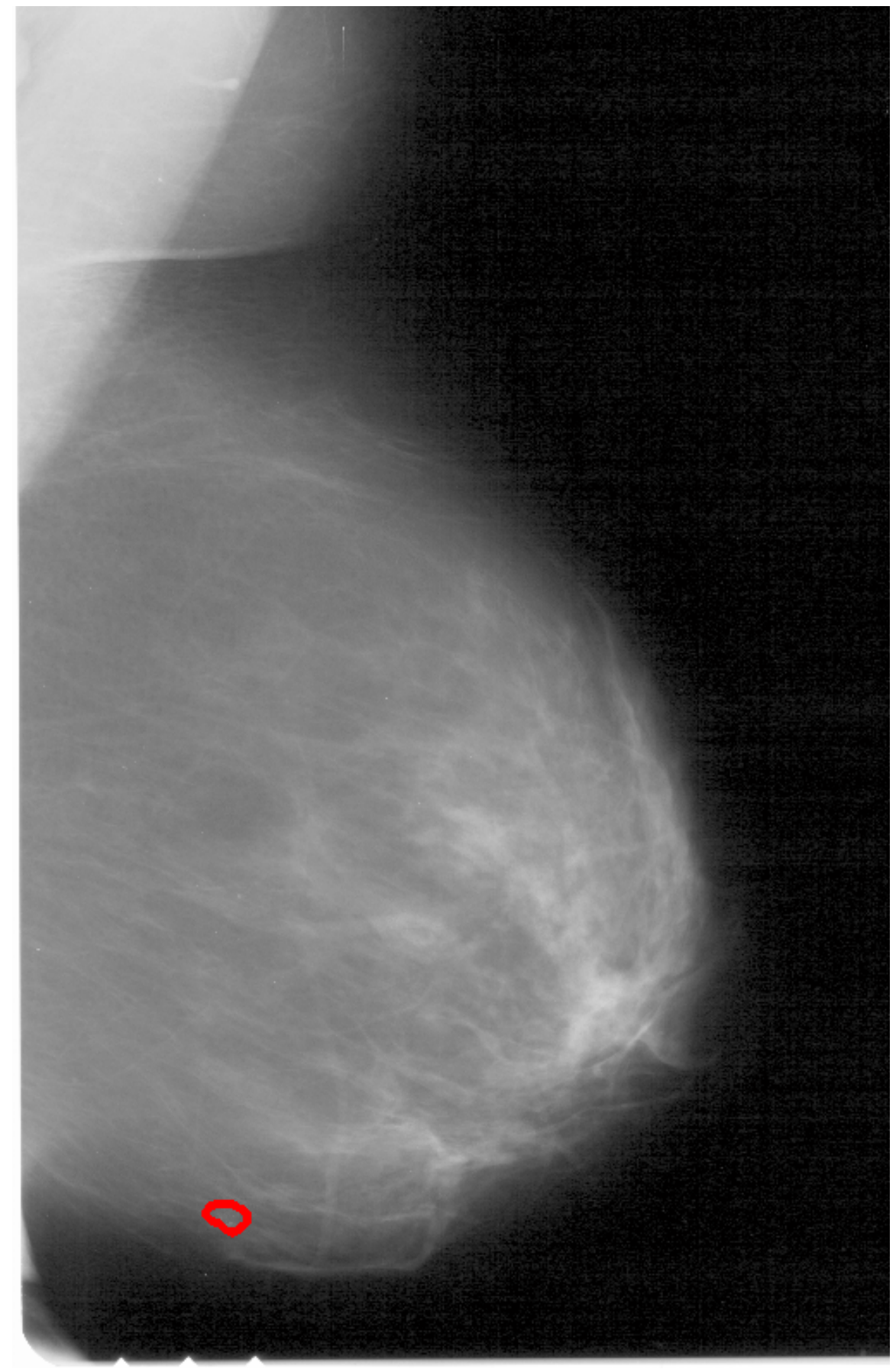

Figure A.1: An example of a DDSM data 


\section{APPENDIX B: MICROCALCIFICATION WITH MAXIMUM $\alpha$ VALUE FOR EACH ROI}

Table B.1: Microcalcification with maximum $\alpha$ value for each ROI

\begin{tabular}{|l|c|c|c|c|}
\hline \multicolumn{1}{|c|}{ DDSM case } & $\alpha$ & Fourier & Moment & Compactness \\
\hline MC_BEN_1259_1.mat & 0.084676431 & 0.025240216 & 0.614769548 & 10.77197715 \\
\hline MC_BEN_1265_4.mat & 0.347223404 & 0.098615428 & 0.490803844 & 6.275492896 \\
\hline MC_BEN_1272_2.mat & 0.19975872 & 0.04252498 & 0.582975705 & 8.810564978 \\
\hline MC_BEN_1275_2.mat & 0.307238554 & 0.128832802 & 0.465227005 & 7.278547845 \\
\hline MC_BEN_1280_2.mat & 0.175864578 & 0.022197345 & 0.598720818 & 9.71375325 \\
\hline MC_BEN_1281_1.mat & 0.237342197 & 0.017690047 & 0.458650294 & 6.095371704 \\
\hline MC_BEN_1285_1.mat & 0.309546224 & 0.037174297 & 0.446486919 & 7.098748289 \\
\hline MC_BEN_1310_1.mat & 0.100753438 & 0.060198211 & 0.598568803 & 8.525565777 \\
\hline MC_BEN_1315_2.mat & 0.266109429 & 0.093868379 & 0.506982529 & 8.664136631 \\
\hline MC_BEN_1316_1.mat & 0.18662573 & 0.091708504 & 0.495537354 & 8.562449088 \\
\hline MC_BEN_1324_1.mat & 0.12187249 & 0.044533998 & 0.542428748 & 12.15427163 \\
\hline MC_BEN_1327_1.mat & 0.098433517 & 0.023290731 & 0.543439737 & 9.419546345 \\
\hline MC_BEN_1328_3.mat & 0.113764708 & 0.041656053 & 0.529854072 & 9.393040294 \\
\hline MC_BEN_1331_1.mat & 0.10741697 & 0.052157479 & 0.58483022 & 8.401850817 \\
\hline MC_BEN_1333_1.mat & 0.120269727 & 0.033295635 & 0.545248786 & 7.796509185 \\
\hline MC_BEN_1345_1.mat & 0.297595852 & 0.178357983 & 0.504386403 & 8.347717089 \\
\hline MC_BEN_1352_2.mat & 0.284108936 & 0.029671215 & 0.53434663 & 10.41572376 \\
\hline MC_BEN_1377_1.mat & 0.04417744 & 0.028339126 & 0.550486705 & 8.186040098 \\
\hline MC_BEN_1378_1.mat & 0.122091168 & 0.044484385 & 0.535612658 & 8.299280465 \\
\hline MC_BEN_1429_1.mat & 0.252155155 & 0.187341983 & 0.461769349 & 8.254601289 \\
\hline MC_BEN_1438_1.mat & 0.084962262 & 0.01535973 & 0.604552061 & 9.9835409 \\
\hline MC_BEN_1441_2.mat & 0.162174241 & 0.031139942 & 0.571004165 & 8.59893698 \\
\hline MC_BEN_1448_5.mat & 0.127118954 & 0.029283897 & 0.61757009 & 10.39044896 \\
\hline MC_BEN_1452_1.mat & 0.20984267 & 0.062868545 & 0.561836721 & 11.12415761 \\
\hline MC_BEN_1458_2.mat & 0.31268102 & 0.054139773 & 0.52432661 & 11.65550049 \\
\hline MC_BEN_1479_1.mat & 0.187935189 & 0.150003775 & 0.376170733 & 3.876132326 \\
\hline MC_BEN_1497_1.mat & 0.1776908 & 0.067601149 & 0.546764185 & 8.030102703 \\
\hline
\end{tabular}


Table B.1 - Continued

\begin{tabular}{|c|c|c|c|c|}
\hline DDSM case & $\alpha$ & Fourier & Moment & Compactness \\
\hline MC_BEN_1513_2.mat & .13194451 & 0.038010922 & 0.602854259 & 10.62193633 \\
\hline MC_BEN_1546_2.mat & 6928 & & 0.58 & \\
\hline MC_BEN_1551_2.mat & 145059977 & 0.12 & 0.48 & \\
\hline MC_BEN_1552_2.mat & .196022898 & 0.086 & 0.436 & \\
\hline MC_BEN_1s & 223 & 0.09 & 0.47 & \\
\hline MC_BEN_16 & $0.282 \xi$ & 0.05 & 0.50 & \\
\hline MC_BEN_1C & 0676 & 0.0 & 0.57 & \\
\hline MC_BEN_1646_2.mat & 0.136648818 & 0.0 & 0.51 & \\
\hline MC_BEN_16 & .299 & 0.0 & 0.52 & \\
\hline MC_BEN_1 & 926 & 0.0 & 0.5 & \\
\hline MC_BEN & 1778 & 0.0 & 0.53 & 86 \\
\hline 1.mat & 114 & & 0.56 & 686 \\
\hline mat & 306 & 0 . & 0.40 & \\
\hline mat & 72 & 31 & 36 & \\
\hline mat & 15 & 77 & 0.5 & 87 \\
\hline mat & 27 & 28 & 28 & \\
\hline nat & 12 & 99 & 0.5 & 83 \\
\hline mat & & & 0.5 & \\
\hline at & & & & \\
\hline MC_BEI & 46 & 0. & 0.5 & \\
\hline C_BEl & 78 & 0.0 & 0.5 & 994 \\
\hline mat & 89 & & 31 & 725 \\
\hline$\overline{\mathrm{C} \_\mathrm{BEI}}$ & 32 & 0.0 & 0.5 & 551 \\
\hline MC_BEI & 26 & 0.0 & 0.52 & 379 \\
\hline MC_BEI & 08 & 0.0 & 0.57 & 838 \\
\hline mat & 43 & & 0.52 & 392 \\
\hline mat & 68 & 0.0 & 0.553 & 0992 \\
\hline mat & 78 & 0.06 & 0.55 & 6723 \\
\hline mat & 463 & 0.02 & 0.530 & 2477 \\
\hline mat & 38 & & 89 & 612 \\
\hline mat & 82 & & 0.600 & \\
\hline at & & & & \\
\hline & & & & \\
\hline MC_BP & 24 & & 0.4267 & \\
\hline
\end{tabular}


Table B.1 - Continued

\begin{tabular}{|c|c|c|c|c|}
\hline case & $\alpha$ & Fourier & Moment & Compactness \\
\hline MC_BEN_1859_1.mat & .097685122 & 0.029189451 & 0.557385169 & 9.253884991 \\
\hline MC_BEN_1864_1.mat & 2891 & 0.253 & 31 & \\
\hline MC_BEN_1866_3.mat & 239783652 & 0.07 & 0.49 & \\
\hline MC_BEN_1867_2.mat & .072810784 & 0.02 & 0.57 & \\
\hline MC_BEN_186 & .212729421 & 0.08 & 0.56 & 177 \\
\hline MC_BEN & .157951025 & 0.0 & 0.5 & \\
\hline MC_BEI & 0.1743 & 0.06 & 0.52 & \\
\hline MC_BEN & .309329762 & 0.09 & 0.43 & \\
\hline$\overline{\mathrm{C} \_\mathrm{BEN}}$ & 0.18982443 & 0.0 & 0.58 & 265 \\
\hline mat & 208772845 & 0.11 & 0.50 & \\
\hline mat & 153849094 & 0.05 & 0.5 & 674 \\
\hline mat & 140 & & 774 & \\
\hline at & 207 & 0.0 & 717 & 379 \\
\hline at & 14 & 0 . & 91 & \\
\hline at & 8 & 45 & 193 & 01 \\
\hline at & .14 & 0.0 & & \\
\hline at & 26 & 0. & 08 & 01 \\
\hline at & 8 & & 25 & 87 \\
\hline at & 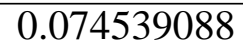 & & & 26 \\
\hline MC_BEI & 68 & & 0.4 & 102 \\
\hline MC_BEI & 37 & 98 & 0.48 & 507 \\
\hline MC_BEI & 77 & 0.0 & 0.48 & \\
\hline MC_BEI & 96 & 0.02 & 0.72 & 467 \\
\hline MC_BEI & 37 & 73 & 0.54 & 55 \\
\hline MC_BEI & $\overline{09}$ & 7 & 0.472 & 7254 \\
\hline MC_BE & 21 & 61 & 0.43 & \\
\hline MC_I & 34 & 0.0 & 0.559 & 4679 \\
\hline at & & 0.06 & 0.53 & \\
\hline lat & 090 & 0.02 & 0.66 & 1733 \\
\hline & 35 & & 0.48 & 2167 \\
\hline at & & & 0.52 & 0189 \\
\hline & & & 0.54 & 437 \\
\hline & & & & \\
\hline MC_BEN & 264145456 & 0.179 & 0.496 & 11.03708282 \\
\hline
\end{tabular}


Table B.1 - Continued

\begin{tabular}{|c|c|c|c|c|}
\hline ase & $\alpha$ & Fourier & Moment & Compactness \\
\hline MC_BEN_400_1.mat & 0.223013595 & 0.0537195 & 0.460600319 & 7.854228687 \\
\hline MC_BEN_409_1.mat & 250726678 & 0.09 & $0.45 \mathrm{~s}$ & \\
\hline MC_BEN_412_2.mat & 163326876 & 707 & 0.47 & \\
\hline MC_BEN_466_1.mat & 191607247 & 0.04 & 0.46 & 663 \\
\hline MC_BEN_476_2.mat & 208673277 & 0.05 & 0.57 & 447 \\
\hline MC_BEN_486_1.mat & 115452647 & 39 & 0.487 & \\
\hline MC_BEN & .225406225 & 0.04 & 0.49 & 743 \\
\hline MC_BEN & .240111809 & 0.02 & 0.47 & 342 \\
\hline MC_BEI & 0.26416649 & 0.0 & 0.46 & 728 \\
\hline C_BEN & .094701262 & 0.0 & 0.52 & 334 \\
\hline BEN & 0008 & $0.0^{\circ}$ & 0.40 & \\
\hline .mat & 045326595 & 0.0 & 0.61 & 669 \\
\hline $\mathrm{BET}$ & .0963 & 73 & 67 & 263 \\
\hline$S E$ & 0.26 & 06 & 45 & 736 \\
\hline $3 \mathrm{E}$ & 559 & 36 & 69 & 304 \\
\hline $\mathrm{N}$ & 3676 & 0 . & 19 & 34 \\
\hline & 96 & & 39 & 941 \\
\hline $\mathrm{N}$ & 93 & & 0.5 & 321 \\
\hline $\mathrm{N}$ & 02 & 39 & & 306 \\
\hline MC_BEN & 276 & & 0.6 & 309 \\
\hline MC_BEN & 4169 & 0.0 & 0.4 & 055 \\
\hline $\mathrm{C} \_\mathrm{BEI}$ & 25 & 44 & 0.5 & 75 \\
\hline MC_BEI & 643 & 0.0 & 0.5 & 453 \\
\hline MC_BEI & 9906 & 0.0 & 0.67 & 382 \\
\hline MC_BEN & 804 & 0.08 & 0.48 & 749 \\
\hline MC_BE & 35 & 0.0 & 0.5 & 519 \\
\hline MC_BEl & 184482442 & 0.02 & 0.56 & 5699 \\
\hline MC_BH & 178689974 & & 050 & \\
\hline 1.mat & 248624834 & 0.02 & 0.502 & 2118 \\
\hline 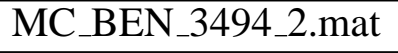 & 1654 & 0.02 & 0.55 & 913 \\
\hline 2.mat & 0.528737747 & 0.37 & 0.36 & 1779 \\
\hline & & & 0.46 & 209 \\
\hline & & & 0.42 & \\
\hline MC_MAL_1153_3 & 0.2841 & 0.08 & 0.526 & 751 \\
\hline
\end{tabular}


Table B.1 - Continued

\begin{tabular}{|c|c|c|c|c|}
\hline ase & $\alpha$ & Fourier & Moment & actness \\
\hline MC_MAL_1185_3.mat & 112192246 & 0.128492554 & 0.467475407 & 9.0448637 \\
\hline MC_MAL_1188_3.mat & 2338 & 0.05 & 0.560 & 169 \\
\hline MC_MAL_1201_3.mat & .317095561 & 0.089999468 & 0.422 & \\
\hline $\mathrm{MC} \_\mathrm{M}$ & 0.256480673 & 0.056049615 & 0.56 & \\
\hline -7.mat & 0.124627097 & 0.02 & 0.509 & \\
\hline MC_N & 0.079253238 & 0.032 & 0.592 & \\
\hline 3.mat & 0.25 & 0.05 & 0.52 & \\
\hline $\mathrm{MC} \_$ & 0.392 & 0.094 & 0.53 & \\
\hline$\overline{\mathrm{MC} \_}$ & 0.4840 & 0.21 & & \\
\hline mat & 0.43324245 & 0.15 & 96 & \\
\hline mat & 0.289252978 & 0.04 & $0.4 \mathrm{C}$ & \\
\hline at & 0.37 & $0.0^{7}$ & & \\
\hline at & 0.30 & & 53 & \\
\hline at & 0.23 & 72 & 49 & \\
\hline at & 44 & 65 & 42 & \\
\hline at & 28 & 0 & 08 & \\
\hline at & 26 & 13 & 0.5 & \\
\hline at & & & 0.4 & \\
\hline at & & & & \\
\hline mat & 59 & 9 & 52 & \\
\hline at & 05 & & 34 & \\
\hline at & 26 & 0.0 & 23 & \\
\hline at & 36 & 0.1 & 0.39 & \\
\hline .mat & 73 & & & 63 \\
\hline .mat & 0.4 & 0.29 & $0.4 \mathrm{C}$ & \\
\hline .mat & 0.04 & 0.02 & 0.60 & \\
\hline mat & 0.338 & 0.35 & 0.369 & \\
\hline mat & 53 & ? & 15 & \\
\hline mat & 0.36 & 0.07 & 0.50 & 071 \\
\hline mat & 0.08 & 0.02 & & 044 \\
\hline mat & & & 0.55 & 85 \\
\hline & & $0.02^{\prime}$ & & \\
\hline & & & & \\
\hline MC_MA & 298070732 & & 0.470328725 & \\
\hline
\end{tabular}


Table B. 1 - Continued

\begin{tabular}{|l|c|c|c|c|}
\hline \multicolumn{1}{|c|}{ DDSM case } & $\alpha$ & Fourier & Moment & Compactness \\
\hline MC_MAL_4182_5.mat & 0.217062366 & 0.030990162 & 0.568507446 & 11.1376256 \\
\hline MC_MAL_63_4.mat & 0.05078913 & 0.039441553 & 0.645653922 & 9.650091481 \\
\hline MC_MAL_87_2.mat & 0.199702352 & 0.067467241 & 0.563289444 & 8.563738456 \\
\hline MC_MAL_90_2.mat & 0.265606749 & 0.098405971 & 0.539460706 & 9.889550053 \\
\hline MC_MAL_96_2.mat & 0.234687875 & 0.074800255 & 0.470739498 & 7.629193722 \\
\hline MC_MAL_99_2.mat & 0.188015136 & 0.137714018 & 0.455260326 & 9.278270858 \\
\hline MC_MAL_106_4.mat & 0.139996976 & 0.044309131 & 0.561431326 & 8.777875486 \\
\hline MC_MAL_132_3.mat & 0.286655134 & 0.053970416 & 0.465514088 & 8.720718134 \\
\hline MC_MAL_167_1.mat & 0.388661871 & 0.456851315 & 0.352259982 & 5.694313149 \\
\hline MC_MAL_171_3.mat & 0.225700483 & 0.077972193 & 0.547883075 & 8.712827494 \\
\hline MC_MAL_309_3.mat & 0.388581978 & 0.05315112 & 0.439518347 & 8.969793882 \\
\hline MC_MAL_3044_4.mat & 0.141011138 & 0.025996265 & 0.556078786 & 7.753603423 \\
\hline MC_MAL_3055_5.mat & 0.199965258 & 0.052269033 & 0.479480094 & 9.188225493 \\
\hline MC_MAL_3382_3.mat & 0.328638051 & 0.122335785 & 0.591837158 & 10.63397466 \\
\hline MC_MAL_3389_2.mat & 0.109308743 & 0.042350634 & 0.626327556 & 9.9927393 \\
\hline MC_MAL_3406_2.mat & 0.357365892 & 0.084558158 & 0.436413 & 9.28687106 \\
\hline MC_MAL_3476_5.mat & 0.346551973 & 0.097383541 & 0.430315119 & 5.791465025 \\
\hline MC_MAL_3498_2.mat & 0.290699714 & 0.091543027 & 0.41481946 & 10.65937887 \\
\hline MC_MAL_3504_2.mat & 0.472145662 & 0.162701336 & 0.504748288 & 9.841025799 \\
\hline MC_MAL_3512_5.mat & 0.089739537 & 0.01109452 & 0.632236507 & 10.01128331 \\
\hline
\end{tabular}




\section{APPENDIX C: TEST RESULT OF ALL MICROCALCIFICATIONS}

Table C.1: Test result of all Microcalcifications

\begin{tabular}{|c|c|c|c|c|}
\hline DDSM case & $\alpha$ & Fourier & Moment & Compactness \\
\hline MC_BEN_1259_1.mat & 4676431 & 0.025240216 & 614769548 & 7715 \\
\hline MC_BEN_1265_4.mat & 0.347223404 & 0.09 & 0.490 & 896 \\
\hline MC_BEN_1265_4.mat & 778 & 516 & 0.4577 & 302 \\
\hline MC_BEN_1265_4.mat & 0.216795795 & 0.042637401 & 0.501735552 & 5181 \\
\hline MC_BEN_1265_4.mat & 0.183348459 & 0.020060262 & 0.548460946 & 4054 \\
\hline MC_BEN_1272_2.mat & 0.19975872 & 2498 & 5705 & 978 \\
\hline MC_BEN_1272_2.mat & 0.168662412 & 0.086794622 & 0.560598761 & 386 \\
\hline MC_BEN_1275_2.mat & 0.307238554 & 0.128832802 & 0.465227005 & 7.2785 \\
\hline MC_BEN_1275_2.mat & 0.223423099 & 0.031963097 & 0.56807436 & 7179 \\
\hline MC_BEN_1280_2.mat & 0.175864578 & 0.022197345 & 0.598720818 & 325 \\
\hline MC_BEN_1280_2 & 24 & 07 & 21 & \\
\hline MC_BEN_1281_1.mat & 0.237342197 & 0.017690047 & 0.458650294 & 6.095371704 \\
\hline MC_BEN_1285_1.mat & 0.309546224 & 0.037174297 & 0.446486919 & 7.098748289 \\
\hline MC_BEN_1310_1.mat & 0.10 & 11 & 0.598568803 & 8.525565777 \\
\hline MC_BEN_1315_2.mat & 0.266109429 & 58379 & 0.506982529 & 8.664136631 \\
\hline MC_BEN_1315_2.mat & 0.287394278 & 0.042281446 & 0.513034326 & 10.44401844 \\
\hline MC_BEN_1316_1.mat & 0.18662573 & 0.091708504 & 0.4955 & 8.562449088 \\
\hline MC_BEN_1324_1.mat & 0.12187249 & 0.044533998 & 0.542428748 & 12.15427163 \\
\hline MC_BEN_1327_1.mat & 0.098433517 & 0.023290731 & 0.543439737 & 9.419546345 \\
\hline MC_BEN_1328_3.mat & 0.113764708 & 0.041656053 & 0.529854072 & 9.393040294 \\
\hline MC_BEN_1328_3.mat & 0.241670388 & 0.162111536 & 0.387854206 & 4.274333802 \\
\hline MC_BEN_1328_3.mat & 0.157785358 & 0.098004272 & 0.533503079 & 9.022604575 \\
\hline MC_BEN_1331_1.mat & 0.10741697 & 0.052157479 & 0.58483022 & 8.401850817 \\
\hline MC_BEN_1333_1.mat & 0.120269727 & 0.033295635 & 0.545248786 & 7.796509185 \\
\hline MC_BEN_1345_1.mat & 0.297595852 & 0.178357983 & 0.504386403 & 8.347717089 \\
\hline MC_BEN_1352_2.mat & 0.284108936 & 0.029671215 & 0.53434663 & 10.41572376 \\
\hline MC_BEN_1352_2.mat & 0.073400738 & 0.02294978 & 0.492543078 & 7.732298033 \\
\hline MC_BEN_1377_1.mat & 0.04417744 & 0.028339126 & 0.550486705 & 8.186040098 \\
\hline MC_BEN_1378_1.mat & 0.122091168 & 0.044484385 & 0.535612658 & 8.299280465 \\
\hline
\end{tabular}


Table C. 1 - Continued

\begin{tabular}{|c|c|c|c|c|}
\hline DDSM case & $\alpha$ & Fourier & Moment & Compactness \\
\hline MC_BEN_1429_1.mat & 0.252155155 & 0.187341983 & 0.461769349 & 8.254601289 \\
\hline MC_BEN_1438_1.mat & 0.084962262 & 0.01535973 & 0.604552061 & 9.9835409 \\
\hline MC_BEN_1441_2.mat & 0.162174241 & 0.031139942 & 0.571004165 & 8.59893698 \\
\hline MC_BEN_1441_2.mat & 0.279243597 & 0.105341045 & 0.561645049 & 10.16309533 \\
\hline MC_BEN_1448_5.mat & 0.127118954 & 0.029283897 & 0.61757009 & 10.39044896 \\
\hline MC_BEN_1448_5.mat & 0.184722411 & 0.075796868 & 0.566136959 & 10.22171802 \\
\hline MC_BEN_1448_5.mat & 0.154813213 & 0.054406874 & 0.502941041 & 7.959480803 \\
\hline MC_BEN_1448_5.mat & 0.155887688 & 0.053570233 & 0.532988734 & 8.104517765 \\
\hline MC_BEN_1448_5.mat & 0.295652852 & 0.090213412 & 0.517314076 & 7.58913176 \\
\hline MC_BEN_1452_1.mat & 0.20984267 & 0.062868545 & 0.561836721 & 11.12415761 \\
\hline MC_BEN_1458_2.mat & 0.31268102 & 0.054139773 & 0.52432661 & 11.65550049 \\
\hline MC_BEN_1458_2.mat & 0.281954769 & 0.036392862 & 0.531580527 & 7.187885731 \\
\hline MC_BEN_1479_1.mat & 0.1 & 75 & 733 & 3.876132326 \\
\hline MC_BEN_1497_1.mat & 0.1776908 & 0.067601149 & 0.546764185 & 8.030102703 \\
\hline MC_BEN_1513_2.mat & 0.13194451 & 0.038010922 & 0.602854259 & 10.62193633 \\
\hline MC_BEN_1513_2.mat & 365471 & 0.051067462 & 0.502229212 & 9.184008586 \\
\hline MC_BEN_1546_2.mat & 0.116896928 & 0.031586494 & 0.589910042 & 8.692571634 \\
\hline MC_BEN_1546_2.mat & 0.156773191 & 0.020678976 & 0.483978334 & 8.245882606 \\
\hline MC_BEN_1551_2.mat & 0.145059977 & 0.142717991 & 0.483688246 & 6.536110046 \\
\hline MC_BEN_1551_2.mat & 0.127551018 & 0.037996084 & 0.541450043 & 8.377847325 \\
\hline MC_BEN_1552_2.mat & 0.196022898 & 0.086496849 & 0.436794415 & 6.898970694 \\
\hline MC_BEN_1552_2.mat & 0.154094774 & 0.103507531 & 0.559176306 & 11.46409708 \\
\hline MC_BEN_1561_1.mat & 0.222197223 & 0.099320461 & 0.478815596 & 11.37333273 \\
\hline MC_BEN_1604_2.mat & 0.282848318 & 0.092711355 & 0.504224402 & 8.893919578 \\
\hline MC_BEN_1604_2.mat & 0.207058997 & 0.113735076 & 0.487965194 & 7.747930085 \\
\hline MC_BEN_1632_1.mat & 0.123430676 & 0.019240017 & 0.573980409 & 6.840128839 \\
\hline MC_BEN_1646_2.mat & 0.136648818 & 0.039310942 & 0.518871612 & 8.350427148 \\
\hline MC_BEN_1646_2.mat & 0.15907701 & 0.147039305 & 0.447876167 & 8.832799424 \\
\hline MC_BEN_1648_2.mat & 0.299679548 & 0.042217244 & 0.527015096 & 8.617010368 \\
\hline MC_BEN_1648_2.mat & 0.149953568 & 0.025059129 & 0.585679818 & 7.75484615 \\
\hline MC_BEN_1649_2.mat & 0.211849926 & 0.043997684 & 0.537139649 & 5.891021974 \\
\hline MC_BEN_1649_2.mat & 0.158438223 & 0.069128332 & 0.596577673 & 10.96006251 \\
\hline MC_BEN_1655_2.mat & 0.176511778 & 0.048719788 & 0.539456337 & 8.295843086 \\
\hline MC_BEN_1655_2.mat & 0.239845366 & 0.062024079 & 0.567465572 & 13.89001336 \\
\hline
\end{tabular}


Table C.1 - Continued

\begin{tabular}{|c|c|c|c|c|}
\hline case & $\alpha$ & Fourier & Moment & Compactness \\
\hline MC_BEN_1735_1.mat & .119490763 & 0.075223955 & 0.569468042 & 9.770666686 \\
\hline MC_BEN_1736_2.mat & .227954306 & & 0.40 & \\
\hline MC_BEN_1736_2.mat & 139333386 & 0.04 & 0.49 & \\
\hline MC_BEN_1740_1.mat & .12047272 & 0.028 & 0.44 & \\
\hline MC_BEN_174 & .116360315 & 0.057894077 & 0.51 & 087 \\
\hline MC_BEN_1743_4.mat & .109720627 & 0.027 & 0.6 & \\
\hline MC_BEN_17 & 0.154046789 & 0.0 & 0.5 & \\
\hline MC_BEN & .203673971 & 0.0 & 0.55 & \\
\hline 4.mat & .183 & 0.0 & 0.52 & \\
\hline -4.mat & .298760412 & 0.16 & 68 & 483 \\
\hline mat & .14087 & & 91 & 988 \\
\hline mat & 09 & 0 . & 21 & 22 \\
\hline mat & 68 & 21 & 56 & 664 \\
\hline nat & 76 & 0 . & 82 & 08 \\
\hline at & 88 & 0.0 & 35 & 563 \\
\hline at & 0.25 & 0 . & 61 & 226 \\
\hline & 46 & 38 & 51 & \\
\hline & 78 & & 03 & 994 \\
\hline & & & & 25 \\
\hline MC_BEI & 32 & & 0.5 & 551 \\
\hline MC_BEI & 26 & 0.0 & 0.5 & 879 \\
\hline $\mathrm{C} \_\mathrm{BEI}$ & 25 & 41 & 0.6 & 485 \\
\hline MC_BEI & 61 & 0.0 & 0.47 & 533 \\
\hline MC_BEI & 08 & 0.0 & 87 & 838 \\
\hline MC_BEl & 43 & 0.0 & 0.52 & 392 \\
\hline MC_BE & 43 & & 0.46 & 187 \\
\hline nat & 8868 & 0.03 & 0.55 & 0992 \\
\hline at & 78 & 0.06 & 0.55 & 6723 \\
\hline at & 463 & 0.02 & 0.530 & 2477 \\
\hline at & 38 & 0.0 & 0.57 & 1612 \\
\hline$\overline{\mathrm{C} B \mathrm{Bl}}$ & 82 & & 0.600 & 238 \\
\hline . & & & & 591 \\
\hline & & & & \\
\hline MC_BP & & 0.01 & 0.54 & 381 \\
\hline
\end{tabular}


Table C.1 - Continued

\begin{tabular}{|c|c|c|c|c|}
\hline DDSM case & $\alpha$ & Fourier & Moment & Compactness \\
\hline MC_BEN_1844_3.mat & .211831534 & 0.097614139 & 0.572290301 & 10.25076383 \\
\hline MC_BEN_1844_3.mat & 139177116 & 0.13 & 0.46 & \\
\hline MC_BEN_1844_3.mat & 150970189 & 0.08 & & \\
\hline MC_BEN_1854_2.mat & .275745524 & 0.10 & 0.42 & 928 \\
\hline MC_BEN_18 & 0.246715265 & 0.05 & 0.50 & \\
\hline MC_BEN_1s & 0.097685122 & 0.02 & 0.55 & \\
\hline MC_BEN_1 & 0.289 & 0.2 & 0.39 & \\
\hline MC_BEN & 0.239783652 & & 0.49 & \\
\hline MC_BEN & 0.1275 & 0.0 & 0.52 & \\
\hline mat & 0.163882378 & 0.1 & 0.51 & \\
\hline mat & 0.07281 & 0.02 & 0.57 & \\
\hline mat & 15 & 0.0 & 04 & 83 \\
\hline lat & 21 & & 59 & 77 \\
\hline at & 22 & & 38 & \\
\hline at & 55 & 91 & 49 & \\
\hline at & 32 & & 29 & 61 \\
\hline & 5 & & & \\
\hline & 4 & & 64 & \\
\hline & & & 18 & \\
\hline MC_BEI & 62 & 0. & 0.4 & 543 \\
\hline$\overline{\mathrm{C} \_\mathrm{BEI}}$ & 51 & 0.0 & 0.46 & 86 \\
\hline at & 58 & 0.0 & 0.3 & 113 \\
\hline MC_BEI & 43 & 0.0 & 283 & 265 \\
\hline MC_BEI & 45 & 28 & 0.50 & \\
\hline MC_BEI & 094 & 0.0 & 0.53 & 674 \\
\hline MC_BE & 14 & 0.0 & 0.47 & \\
\hline MC_BE & 2074 & 0.08 & 5566 & 28797 \\
\hline at & 68 & 0.09 & 0.52 & \\
\hline 1.mat & 14 & 0.11 & 0.531 & \\
\hline at & & & 0.535 & \\
\hline nat & 84 & & 0.50 & 416 \\
\hline $\mathrm{dl}$ & 94 & & 0.327 & 918 \\
\hline & & & & \\
\hline MC_BEN & 137058478 & 0.03 & 0.55 & 8204 \\
\hline
\end{tabular}


Table C. 1 - Continued

\begin{tabular}{|c|c|c|c|c|}
\hline DDSM case & $\alpha$ & Fourier & Moment & Compactness \\
\hline MC_BEN_1935_2.mat & 0.122161088 & 0.032322241 & 0.568010725 & 7.527438287 \\
\hline MC_BEN_1935_2.mat & 0.176768781 & 0.05336069 & 0.523683099 & 10.05813958 \\
\hline MC_BEN_1944_2.mat & 0.074539088 & 0.021776604 & 0.581924399 & 4.700994026 \\
\hline MC_BEN_1944_2.mat & 0.225042001 & 0.028798321 & 0.561924346 & 6.657550062 \\
\hline MC_BEN_1945_2.mat & 0.235715368 & 0.052547324 & 0.478283253 & 11.95492102 \\
\hline MC_BEN_1945_2.mat & 0.25563091 & 0.063607192 & 0.433109195 & 7.273062918 \\
\hline MC_BEN_1949_1.mat & 0.18349787 & 0.133491298 & 0.489285859 & 8.177304507 \\
\hline MC_BEN_1953_1.mat & 0.148433677 & 0.047074708 & 0.489683071 & 9.87323803 \\
\hline MC_BEN_236_1.mat & 0.03611096 & 0.026877529 & 0.721452799 & 11.65276467 \\
\hline MC_BEN_242_1.mat & 0.215991537 & 0.09482873 & 0.547788832 & 11.32481955 \\
\hline MC_BEN_272_2.mat & 0.19285309 & 0.04664157 & 0.472461752 & 6.626807254 \\
\hline MC_BEN_272_2.mat & 0.133565826 & 0.058062166 & 0.415959654 & 4.980920643 \\
\hline MC_BEN_281_1.mat & 0.256007421 & 061 & 0.437578831 & 7.31377138 \\
\hline MC_BEN_300_1.mat & 0.080088734 & 0.030952231 & 0.559494298 & 8.241624679 \\
\hline MC_BEN_301_2.mat & 0.122658959 & 0.060489579 & 0.53672005 & 9.853453993 \\
\hline MC_BEN_301_2.mat & 0.122658959 & 0.060489579 & 0.53672005 & 9.853453993 \\
\hline MC_BEN_307_6.mat & 0.090573327 & 0.02651801 & 0.662553259 & 11.07401733 \\
\hline MC_BEN_307_6.mat & 0.091385275 & 0.036510017 & 0.588151702 & 11.20120709 \\
\hline MC_BEN_307_6.mat & 0.122065266 & 0.065240284 & 0.554541442 & 10.35081242 \\
\hline MC_BEN_307_6.mat & 0.184919261 & 0.039458613 & 0.5415229 & 9.699468874 \\
\hline MC_BEN_315_1.mat & 0.275655885 & 0.073929006 & 0.483081529 & 8.910022167 \\
\hline MC_BEN_375_3.mat & 0.132834731 & 0.036762227 & 0.524491058 & 7.367340189 \\
\hline MC_BEN_375_3.mat & 0.218686562 & 0.063129618 & 0.523975958 & 10.63535362 \\
\hline MC_BEN_375_3.mat & 0.197721243 & 0.048412198 & 0.557123438 & 10.76963788 \\
\hline MC_BEN_379_1.mat & 0.087574698 & 0.020663597 & 0.545480485 & 9.252053437 \\
\hline MC_BEN_390_2.mat & 0.171372115 & 0.050093408 & 0.556085569 & 10.84750429 \\
\hline MC_BEN_390_2.mat & 0.190459437 & 0.040789942 & 0.551022548 & 9.453572471 \\
\hline MC_BEN_391_2.mat & 0.264145456 & 0.179927615 & 0.496953644 & 11.03708282 \\
\hline MC_BEN_391_2.mat & 0.186536385 & 0.046829194 & 0.543529582 & 10.43013586 \\
\hline MC_BEN_400_1.mat & 0.223013595 & 0.0537195 & 0.460600319 & 7.854228687 \\
\hline MC_BEN_409_1.mat & 0.250726678 & 0.090103446 & 0.459862987 & 8.575346769 \\
\hline MC_BEN_412_2.mat & 0.163326876 & 0.11976707 & 0.476834382 & 6.459242578 \\
\hline MC_BEN_412_2.mat & 0.200445295 & 0.073989382 & 0.459862296 & 10.12313383 \\
\hline MC_BEN_466_1.mat & 0.191607247 & 0.044737435 & 0.464235692 & 8.972168663 \\
\hline
\end{tabular}


Table C.1 - Continued

\begin{tabular}{|c|c|c|c|c|}
\hline DDSM case & $\alpha$ & Fourier & Moment & Compactness \\
\hline MC_BEN_476_2.mat & 0.208673277 & 0.057665175 & 0.573991942 & 155447 \\
\hline MC_BEN_476_2.mat & .24646867 & 0.09 & 0.47 & \\
\hline MC_BEN_486_1.mat & 115452647 & 0.0 & 0.487 & \\
\hline MC_BEN_486_1.mat & .225406225 & 0.049 & 0.499 & \\
\hline MC_BEN_492_1.mat & .240111809 & 0.024398173 & 0.47 & \\
\hline MC_BEN_500_1.mat & 0.26416649 & 0.08 & 0.469 & 728 \\
\hline MC_BEN_5 & .094701262 & 0.05 & 0.52 & \\
\hline MC_BEN_502_2.mat & 0.21031948 & 0.0 & 0.52 & 399 \\
\hline MC_BEN_5 & 0008 & $0.0^{\prime}$ & 0.40 & \\
\hline MC_BEN_5( & 248606286 & 0.1 & 0.34 & 303 \\
\hline MC_BEN_3 & 0.045326595 & 0.01 & 0.61 & 669 \\
\hline MC_BEN_ & .096328372 & 0.0 & 67 & 263 \\
\hline 3.mat & 0.13 & 0.0 & 44 & \\
\hline 3.mat & .227 & 0. & 91 & 125 \\
\hline$t$ & 23 & & 45 & 736 \\
\hline at & 59 & & 0.4 & 304 \\
\hline $\mathrm{N}$ & 76 & & 19 & \\
\hline 1. & 6 & & 0.4 & \\
\hline & & & & \\
\hline MC_BEN & 02 & 0.0 & 0.5 & 306 \\
\hline C_BEI & 3276 & & 0.6 & 309 \\
\hline $\mathrm{C} \_\mathrm{BEI}$ & 69 & 0 & 98 & 55 \\
\hline C_BEI & 25 & 0.0 & 0.5 & 175 \\
\hline MC_BEI & 43 & 0. & 2.5 & 453 \\
\hline MC_BEN & 06 & 0.0 & 0.67 & 3382 \\
\hline C_BE & 04 & 0.0 & 0.48 & 749 \\
\hline 2.mat & 35 & 0.04 & 0.558 & 519 \\
\hline $2 . n$ & 92 & 0.1 & 0.44 & 743 \\
\hline 1.mat & 184482442 & $0.0^{2}$ & 0.56 & 5699 \\
\hline 3.1 & 974 & & 0.50 & \\
\hline $3 . n$ & 997 & 0.10 & 0.54 & 078 \\
\hline $6 \_3.1$ & & & & 346 \\
\hline & & & & \\
\hline MC_BP & & 0.02 & 0.55 & \\
\hline
\end{tabular}


Table C. 1 - Continued

\begin{tabular}{|c|c|c|c|c|}
\hline DDSM case & $\alpha$ & Fourier & Moment & Compactness \\
\hline MC_BEN_3494_2.mat & 0.155505113 & 0.034026408 & 0.492395738 & 10.66144207 \\
\hline MC_MAL_1133_2.mat & 0.528737747 & 0.377447031 & 0.36383988 & 4.845661779 \\
\hline MC_MAL_1133_2.mat & 0.345500653 & 0.088929271 & 0.439578153 & 12.94972812 \\
\hline MC_MAL_1148_3.mat & 0.33041194 & 0.149666687 & 0.469996884 & 11.05426209 \\
\hline MC_MAL_1148_3.mat & 0.282804711 & 0.151408563 & 0.481554818 & 8.532901089 \\
\hline MC_MAL_1148_3.mat & 0.181621259 & 0.064488477 & 0.508974985 & 6.956158918 \\
\hline MC_MAL_1152_4.mat & 0.172003283 & 0.052078644 & 0.426732332 & 7.994123988 \\
\hline MC_MAL_1152_4.mat & 0.216414992 & 0.10098766 & 0.432927446 & 7.077399794 \\
\hline MC_MAL_1152_4.mat & 0.455556123 & 0.078908976 & 0.430316652 & 7.60142201 \\
\hline MC_MAL_1152_4.mat & 0.222613356 & 0.023175378 & 0.564635592 & 7.180828656 \\
\hline MC_MAL_1153_3.mat & 0.28414292 & 0.083882634 & 0.52621936 & 9.143124751 \\
\hline MC_MAL_1153_3.mat & 0.148640108 & 0.056693612 & 0.477384507 & 6.936419176 \\
\hline MC_MAL_1185_3.mat & 246 & 554 & 0.467475407 & 9.0448637 \\
\hline MC_MAL_1185_3.mat & 0.191160486 & 0.11644072 & 0.5612425 & 9.041645597 \\
\hline MC_MAL_1185_3.mat & 0.422788998 & 0.060199006 & 0.536082378 & 9.744836693 \\
\hline MC_MAL_1188_3.mat & 0.291342338 & 0.055304239 & 0.560161147 & 12.09495169 \\
\hline MC_MAL_1188_3.mat & 0.355078026 & 0.191564467 & 0.522751453 & 10.92719035 \\
\hline MC_MAL_1188_3.mat & 0.319565169 & 0.063210004 & 0.570607285 & 10.94244664 \\
\hline MC_MAL_1201_3.mat & 0.317095561 & 0.089999468 & 0.422986739 & 6.240750261 \\
\hline MC_MAL_1201_3.mat & 0.237329422 & 0.103454395 & 0.428335636 & 7.023826361 \\
\hline MC_MAL_1201_3.mat & 0.12478339 & 0.05777666 & 0.539548369 & 6.745810699 \\
\hline MC_MAL_1212_2.mat & 0.256480673 & 0.056049615 & 0.561035336 & 10.79214147 \\
\hline MC_MAL_1212_2.mat & 0.207005331 & 0.056406896 & 0.493295602 & 11.56877473 \\
\hline MC_MAL_1213_7.mat & 0.124627097 & 0.02417126 & 0.509627126 & 6.363471119 \\
\hline MC_MAL_1213_7.mat & 0.17518174 & 0.074580629 & 0.52457311 & 9.317780736 \\
\hline MC_MAL_1213_7.mat & 0.451484839 & 0.140444439 & 0.469012625 & 7.66219232 \\
\hline MC_MAL_1213_7.mat & 0.209321493 & 0.0501448 & 0.425808854 & 8.441036208 \\
\hline MC_MAL_1213_7.mat & 0.284478239 & 0.076442209 & 0.472535592 & 8.233720437 \\
\hline MC_MAL_1213_7.mat & 0.268503243 & 0.053629271 & 0.450112581 & 6.541356035 \\
\hline MC_MAL_1213_7.mat & 0.375283767 & 0.10293157 & 0.372789888 & 2.975391114 \\
\hline MC_MAL_1214_6.mat & 0.079253238 & 0.032063042 & 0.592798761 & 9.791501017 \\
\hline MC_MAL_1214_6.mat & 0.39059576 & 0.143260547 & 0.44571901 & 7.0591608 \\
\hline MC_MAL_1214_6.mat & 0.339051086 & 0.09554589 & 0.497510121 & 10.09102722 \\
\hline MC_MAL_1214_6.mat & 0.103350291 & 0.03460591 & 0.591183777 & 9.394354864 \\
\hline
\end{tabular}


Table C.1 - Continued

\begin{tabular}{|c|c|c|c|c|}
\hline case & $\alpha$ & Fourier & Moment & Compactness \\
\hline MC_MAL_1214_6.mat & $\overline{0135}$ & 0.078774046 & 0.519117524 & 8.414158555 \\
\hline MC_MAL_1214_6.mat & 0.36603128 & 83 & & \\
\hline MC_MAL_1223_3.mat & .255613256 & 0.05 & 0.527 & \\
\hline MC_MAL_1223_3.mat & 235853133 & 0.248 & 0.40 & 882 \\
\hline MC_MAL_1235_3.mat & 0.39296243 & 0.094 & 0.53 & \\
\hline $\mathrm{MC} \_\mathrm{M}$ & .208009425 & 0.07 & 0.57 & \\
\hline 1238_2.mat & .484097037 & 0.21 & 0.4 & \\
\hline 2.mat & .20074468 & 0.02 & 0.46 & \\
\hline 2.mat & 43324245 & 0.1 & 0.53 & \\
\hline & .28321241 & 0.04 & 0.44 & 299 \\
\hline & .289252978 & 0.04 & 0.4 & 768 \\
\hline & 269 & 0 . & 48 & \\
\hline & 080 & 0 . & 54 & 628 \\
\hline & 29 & 56 & & \\
\hline & .086 & 17 & 63 & 567 \\
\hline & 44 & 61 & 35 & 39 \\
\hline & & & & 106 \\
\hline & 93 & 68 & 0.5 & 785 \\
\hline & & & & 374 \\
\hline $\mathrm{MC}$ & .20 & 0.0 & 0.4 & 741 \\
\hline MC & 304 & 7 & 0.4 & \\
\hline $\mathrm{M}$ & 26 & & & 75 \\
\hline MC & 2340 & 72 & 0.54 & 065 \\
\hline $\mathrm{MC}$ & 4096 & 0.27 & 0.4 & 185 \\
\hline $\mathrm{MC}$ & .254609044 & 0.12 & 0.52 & 751 \\
\hline $\mathrm{MC}$ & .16910 & 0.0 & 804 & 2574 \\
\hline mat & 263603928 & 0.05 & $0.49^{\prime}$ & 9908 \\
\hline mat & 12 & 0.10 & 0.47 & 9903 \\
\hline 3.mat & 0.526 & 0.15 & 0.457 & 6281 \\
\hline mat & 26 & 0.08 & 0.53 & 809 \\
\hline & 0.456 & & 0.490 & 401 \\
\hline & & & 13 & 937 \\
\hline & & & & \\
\hline MC_MAL_ & & 0.070 & 0.402003621 & 3083 \\
\hline
\end{tabular}


Table C.1 - Continued

\begin{tabular}{|c|c|c|c|c|}
\hline case & $\alpha$ & Fourier & Moment & Compactness \\
\hline MC_MAL_1528_3.mat & 306801029 & 0.058848948 & 0.409957973 & 4.200789478 \\
\hline MC_MAL_1528_3.mat & 3086 & & & \\
\hline MC_MAL_1531_3.mat & 319234481 & 0.087 & & \\
\hline MC_MA & .152708705 & 0.0 & 0.53 & \\
\hline MC_MA & .078910016 & & 0.58 & \\
\hline $\mathrm{MC} \_\mathrm{M} A$ & .383554559 & 0.1 & 0.45 & \\
\hline 2.mat & .237 & 0.02 & 0.5 & \\
\hline )_1.mat & 4405 & 0.13 & 0.44 & \\
\hline 3.mat & 3726 & & 0.48 & \\
\hline mat & 7093 & 0.2 & 283 & \\
\hline mat & 1228 & 0.0 & 288 & \\
\hline mat & 3126 & 0.1 & 11 & 268 \\
\hline mat & 279 & & 876 & \\
\hline mat & 343 & & & 63 \\
\hline mat & 95 & 09 & 004 & 349 \\
\hline mat & 9 & 16 & 29 & 31 \\
\hline mat & & & 86 & \\
\hline mat & 9 & & & \\
\hline mat & 1 & & & \\
\hline mat & 46 & 0. & 0.5 & 262 \\
\hline mat & 09 & & 0.6 & 499 \\
\hline mat & 05 & & 0.6 & 718 \\
\hline mat & 2399 & 0.0 & 0.5 & 406 \\
\hline $\mathrm{MC}$ & 338 & & 0.36 & 831 \\
\hline $\mathrm{MC}$ & .29 & 0.1 & 0.55 & 5853 \\
\hline mat & 30 & 0.0 & 0.47 & \\
\hline mat & .315 & 0.09 & 0.417 & 331 \\
\hline mat & .08 & 0.0 & 26 & 821 \\
\hline mat & 0.16 & 0.04 & 0.57 & 386 \\
\hline mat & & & 0.50 & 071 \\
\hline mat & 0.08 & 0.02 & 0.57 & $\overline{044}$ \\
\hline inat & & & 971 & \\
\hline & & & & \\
\hline MC_MAL_ & & 0.06 & 0.47 & 829 \\
\hline
\end{tabular}


Table C. 1 - Continued

\begin{tabular}{|c|c|c|c|c|}
\hline DDSM case & $\alpha$ & Fourier & Moment & Compactness \\
\hline MC_MAL_4151_5.mat & 0.280896199 & 0.060216824 & 0.483313692 & 10.0384325 \\
\hline MC_MAL_4151_5.mat & 0.10782223 & 0.060044194 & 0.57394912 & 8.446726407 \\
\hline MC_MAL_4151_5.mat & 0.16972228 & 0.081322371 & 0.580107609 & 10.20379928 \\
\hline MC_MAL_4117_2.mat & 0.252391081 & 0.027172212 & 0.427275323 & 04505 \\
\hline MC_MAL_4117_2.mat & 0.201221691 & 0.042755448 & 0.613683593 & 10.58656446 \\
\hline MC_MAL_4158_1.mat & 0.272765728 & 0.188414977 & 0.393244502 & 4.642228102 \\
\hline MC_MAL_4161_2.mat & 0.298070732 & 0.06641729 & 0.470328725 & 6.944532205 \\
\hline MC_MAL_4161_2.mat & 0.539178946 & 0.081682414 & 0.531691923 & 8.6697808 \\
\hline MC_MAL_4182_5.mat & 0.217062366 & 0.030990162 & 0.568507446 & 11.1376256 \\
\hline MC_MAL_4182_5.mat & 0.346165367 & 0.077566573 & 0.409462604 & 5.743061337 \\
\hline MC_MAL_4182_5.mat & 0.151828105 & 0.075565922 & 0.539607809 & 10.04242461 \\
\hline MC_MAL_4182_5.mat & 0.280567924 & 0.07313492 & 0.512680973 & 8.846371331 \\
\hline MC_MAL_4182_5.mat & 0.13901 & 0.05232 & 0.535740904 & 10.66775184 \\
\hline MC_MAL_63_4.mat & 0.05078913 & 0.039441553 & 0.645653922 & 9.650091481 \\
\hline MC_MAL_63_4.mat & 0.069732072 & 0.01350984 & 0.625159677 & 10.48653801 \\
\hline MC_MAL_63_4.mat & 0.075639336 & 0.073502706 & 0.605787149 & 10.85134788 \\
\hline MC_MAL_63_4.mat & 0.322387299 & 0.059693762 & 0.581634993 & 12.70595006 \\
\hline MC_MAL_87_2.mat & 0.199702352 & 0.067467241 & 0.563289444 & 8.563738456 \\
\hline MC_MAL_87_2.mat & 0.361577435 & 0.064740406 & 0.467311351 & 11.75524669 \\
\hline MC_MAL_90_2.mat & 0.265606749 & 0.098405971 & 0.539460706 & 9.889550053 \\
\hline MC_MAL_90_2.mat & 0.092386819 & 0.031623253 & 0.574672448 & 10.54962524 \\
\hline MC_MAL_96_2.mat & 0.234687875 & 0.074800255 & 0.470739498 & 7.629193722 \\
\hline MC_MAL_96_2.mat & 0.421763241 & 0.16296421 & 0.415408277 & 7.070163511 \\
\hline MC_MAL_99_2.mat & 0.188015136 & 0.137714018 & 0.455260326 & 9.278270858 \\
\hline MC_MAL_99_2.mat & 0.503667296 & 0.22729483 & 0.378263597 & 5.786918791 \\
\hline MC_MAL_106_4.mat & 0.139996976 & 0.044309131 & 0.561431326 & 8.777875486 \\
\hline MC_MAL_106_4.mat & 0.468983351 & 0.127951778 & 0.500079687 & 7.853323192 \\
\hline MC_MAL_106_4.mat & 0.251099422 & 0.071029401 & 0.545608669 & 10.77008865 \\
\hline MC_MAL_106_4.mat & 0.20445071 & 0.060898494 & 0.454290225 & 5.98186467 \\
\hline MC_MAL_132_3.mat & 0.286655134 & 0.053970416 & 0.465514088 & 8.720718134 \\
\hline MC_MAL_132_3.mat & 0.211367938 & 0.104350373 & 0.551084621 & 6.888984272 \\
\hline MC_MAL_132_3.mat & 0.111090457 & 0.060799648 & 0.541949396 & 10.65605139 \\
\hline MC_MAL_167_1.mat & 0.388661871 & 0.456851315 & 0.352259982 & 5.694313149 \\
\hline MC_MAL_171_3.mat & 0.225700483 & 0.077972193 & 0.547883075 & 8.712827494 \\
\hline
\end{tabular}


Table C.1 - Continued

\begin{tabular}{|c|c|c|c|c|}
\hline se & $\alpha$ & er & Moment & Compactness \\
\hline MC_MAL & 2475 & & & \\
\hline 6 & & & & \\
\hline & & & & \\
\hline & & & & \\
\hline & & & & \\
\hline & & & & \\
\hline & 1 & & & \\
\hline & & & & \\
\hline & & & & \\
\hline & 8 & & 94 & \\
\hline & & & & \\
\hline & & & 58 & \\
\hline & & & & \\
\hline & & & & \\
\hline & & & & \\
\hline mat & 7 & & & 273 \\
\hline mat & 43 & & 56 & \\
\hline mat & & & 11 & \\
\hline & & & & \\
\hline $\mathrm{MC}$ & & & 43 & \\
\hline & 73 & & 19 & \\
\hline & 72 & & 15 & \\
\hline iat & 09 & 04 & 0.42 & \\
\hline at & 04 & 48 & 79 & 616 \\
\hline mat & 156 & 0.1 & 0.42 & \\
\hline .mat & 14 & 0.0 & 0.4 & 887 \\
\hline$\overline{\mathrm{C} \_\mathrm{MAI}}$ & 238030455 & 123 & 364 & 4547 \\
\hline at & 662 & 36 & 288 & 799 \\
\hline 2.mat & 11 & 553 & 06 & 035 \\
\hline & 537 & & 07 & \\
\hline at & 75 & 44 & 46 & 041 \\
\hline & & & & \\
\hline MC_MAL_? & 526 & 0. & & \\
\hline
\end{tabular}




\section{BIBLIOGRAPHY}

[1] H. Soltanian-Zadeh, F. Rafiee-Rad, and S. Pourabdollah-Nejad D, "Comparison of multiwavelet, wavelet, haralick, and shape features for microcalcification classification in mammograms," Pattern Recognition, vol. 37, no. 10, pp. 1973 - 1986, 2004.

[2] “Deaths: Final data for 2006," National Vital Statistics Reports, vol. 57, no. 14, pp. $1-135,2009$.

[3] “Breast cancer facts \& figures 2009-2010," http://www.cancer.org/downloads/STT/ F861009_final\%209-08-09.pdf, 2009 (accessed Feb 6, 2010).

[4] "Breast cancer: Early detection," http://www.cancer.org/docroot/CRI/content/ CRI_2_6x_Breast_Cancer_Early_Detection.asp, 2009 (accessed Feb 6, 2010).

[5] E. A. Sickles, "Breast calcifications: Mammographic evaluation," Radiology, vol. 160, pp. 289-293, 1986.

[6] H. D. Cheng, X. Cai, X. Chen, L. Hu, and X. Lou, "Computer-aided detection and classification of microcalcifications in mammograms: a survey," Pattern Recognition, vol. 36, no. 12, pp. 2967 - 2991, 2003.

[7] F. Lefebvre, H. Benali, E. Kahn, and R.D. Paola, "A fractal approach tothe segmentation of microcalcifications in digital mammograms," Med.Phys., vol. 22, no. 4, pp. 381-391, 1995. 
[8] N. S. Arikidis, A. Karahaliou, S. Skiadopoulos, P.s Korfiatis, E. Likaki, G. Panayiotakis, and L. Costaridou, "Size-adapted microcalcification segmentation in mammography utilizing scale-space signatures," Computerized Medical Imaging and Graphics, vol. In Press, Corrected Proof, pp. -, 2010.

[9] L. P. Clarke, M. Kallergi, W. Qian, H. Li, R. A. Clark, and S. M. L., “Tree-structured non-linear filter and wavelet transform for microcalcification segmentation in digital mammography," Cancer Letters, vol. 77, no. 2-3, pp. 173 - 181, 1994, Computer applications for early detection and staging of cancer.

[10] B. Liu, H.D. Cheng, J. Huang, J. Tian, J. Liu, and X. Tang, “Automated segmentation of ultrasonic breast lesions using statistical texture classification and active contour based on probability distance," Ultrasound in Medicine \& Biology, vol. 35, no. 8, pp. $1309-1324,2009$.

[11] A. Papadopoulos, D. I. Fotiadis, and A. Likas, "An automatic microcalcification detection system based on a hybrid neural network classifier," Artificial Intelligence in Medicine, vol. 25, no. 2, pp. 149 - 167, 2002.

[12] M. G. Linguraru, K. Marias, R. English, and M. Brady, "A biologically inspired algorithm for microcalcification cluster detection," Medical Image Analysis, vol. 10, no. 6 , pp. $850-862,2006$.

[13] A. Papadopoulos, D.I. Fotiadis, and L. Costaridou, "Improvement of microcalcification cluster detection in mammography utilizing image enhancement techniques," Computers in Biology and Medicine, vol. 38, no. 10, pp. 1045 - 1055, 2008.

[14] N.a Ahuja, A. Rosenfeld, and R. M. Haralick, "Neighbor gray levels as features in pixel classification," Pattern Recognition, vol. 12, no. 4, pp. 251 - 260, 1980. 
[15] R. M. Haralick, "Statistical and structural approaches to texture," Proc. IEEE, vol. 67, no. 5, pp. $786-804,1979$.

[16] J.Y. Tham, L.X. Shen, S.L. Lee, and H.H. Tan, “A general approach for analysis and application of discrete multiwavelet transforms," Signal Processing, vol. 48, no. 2, pp. $457-464,2000$.

[17] S. Yu, K. Li, and Y. Huang, "Detection of microcallcification in digital mammograms using wavelet filter and markov random field model," Computerized Medical Imaging and Graphics, vol. 30, pp. 163 - 173, 2006.

[18] L. Shen, R. M. Rangayyan, and J. E. L. Desautels, "Application of shape analysis to mammographic calcifications," IEEE Trans. Med. Imag., vol. 13, no. 2, pp. 263-274, June 1994.

[19] C. Xu and J. L. Prince, "Snakes, shapes, and gradient vector flow," IEEE Trans. Image Processing, vol. 7, no. 3, pp. 359-369, March 1998.

[20] M. Kass, A. Witkin, and D. Terzopoulos, "Snakes: Active contour models," Int. J. Comput. Vis., vol. 1, pp. 321 - 331, 1987.

[21] R. C. Gonzalez and R. E. Woods, Digital Image Processing, Prentice Hall, Upper Saddle River, New Jersey 07458, second edition, 2002.

[22] T. Fawcett, "An introduction to roc analysis," Pattern Recognition Letters, vol. 27, no. 8 , pp. 861 - 874, 2006, ROC Analysis in Pattern Recognition. 